\title{
Abstracts From the December 2019 International Sport + Exercise Nutrition Conference in Newscastle upon Tyne
}

\section{Day 1 Orals: Performance Theme}

\section{Tuesday, 17th December 2019}

\author{
14.00 \\ Dietary intake of Gaelic football players during game preparation and \\ recovery \\ C Ó Catháin, J Fleming, M Renard, D Kelly

\subsection{5} \\ Enriching a lower dose of mycoprotein with branched chain amino \\ acids does not stimulate protein synthesis rates to the same extent as a \\ higher dose of mycoprotein in rested and exercised skeletal muscle of \\ healthy young men
}

AJ Monteyne, MO Coelho, TSO Jameson, C Porter, DR Abdelrahman, TJA Finnigan, ML Dirks, FB Stephens, BT Wall

\subsection{0}

Equation based on anthropometry to predict body fat percentage by DXA in elite youth soccer athletes

A Batista, D Granja, R Borrego, E Carolino, C Caetano

14.45

Sports nutrition knowledge of Gaelic football players

M Renard, A Anton-Solanas, D Kelly, C Ó Catháin

$\mathbf{1 5 . 0 0}$

New equation to estimate body fat mass in international-elite soccer referees

C Petri, VH Teixeira, P Izzicupo, G Galanti, A Pizzi, G Mascherini

\section{$\mathbf{1 5 . 1 5}$}

Is it necessary to ingest carbohydrates and fluids during $10 \mathrm{~km}$ open water swimming in top-level competitions?

G Olcina, R Timón, I González-Pérez.

Dietary Intake of Gaelic Football Players During Game Preparation and Recovery

C Ó Catháin ${ }^{1}$, J Fleming ${ }^{2}$, M Renard ${ }^{1}$, D Kelly ${ }^{1}$

${ }^{1}$ Department of Sport and Health Sciences, Athlone Institute of Technology, Ireland

${ }^{2}$ Department of Health Sciences, St Mary's University, Twickenham, London, UK

It is well established that dietary intake can influence performance and modulate recovery in invasion-based team sports such as soccer, and rugby. However, very limited research currently exists examining dietary intake of Gaelic football players. This research aimed to examine the dietary intake of Gaelic football player's 2-days prior to competition, on game day, and for 2-days post competition in order to assess if 1) player's intake is in line with current sports nutrition recommendations; 2) if intake varies from day to day, and; 3) if playing level (Elite Vs. Sub-elite) influences intake. A five-day paper-based food diary was completed by 45 players ( 25 elite and 20 sub-elite). Playing level had no effect on energy, carbohydrate, or fat intake, but elite players consumed $24 \%$ more protein than sub-elite. Average intake of energy was $2938 \pm 618 \mathrm{kcal}^{-d a y}{ }^{-1}$, carbohydrate was $3.7 \pm 1.4 \mathrm{~g} . \mathrm{kg} \mathrm{bm}^{-1}$. day ${ }^{-1}$, and fat was $1.3 \pm 0.6 \mathrm{~g} . \mathrm{kg}$ $\mathrm{bm}^{-1}$.day ${ }^{-1}$. Elite players consumed $2.2 \pm 0.7 \mathrm{~g} . \mathrm{kg} \mathrm{bm}^{-1}$.day ${ }^{-1}$ protein, whereas sub elite consumed $1.8 \pm 0.6 \mathrm{~g} \cdot \mathrm{kgbm}^{-1}$. day ${ }^{-1}$. Day-to-day variation in carbohydrate intake was apparent (Day 1 (Training) $>$ Day 5 (Training) by $21 \%$; Day 3 (Game day) > Day 5 by $29 \%$ ), but this did not take place in a manner that reflected purposeful nutritional periodization. Regardless of playing level, players consumed inadequate amounts of carbohydrate to support optimal performance and recovery and consumed protein and fat in line with general sport nutrition guidelines. However, given the unique demands placed on Gaelic football players, it may be necessary to develop Gaelic Football specific nutrition guidelines. Despite this, to address the displayed nutritional inadequacies, it is necessary to design and implement Gaelic football specific nutrition education-based interventions.

Enriching a Lower Dose of Mycoprotein With Branched Chain Amino Acids Does not Stimulate Protein Synthesis Rates to the Same Extent as a Higher Dose of Mycoprotein in Rested and Exercised Skeletal Muscle of Healthy Young Men

AJ Monteyne ${ }^{1}$, MO Coelho ${ }^{1}$, TSO Jameson ${ }^{1}$, C Porter $^{2,3}$, DR Abdelrahman ${ }^{2}$, TJA Finnigan ${ }^{4}$, ML Dirks $^{1}$, FB Stephens ${ }^{1}$, BT Wall ${ }^{1}$

${ }^{1}$ Nutritional Physiology Research Group, Sport and Health Sciences, University of Exeter, UK

${ }^{2}$ Department of Pediatrics, University of Arkansas for Medical Sciences, Arkansas Children's Research Institute, Arkansas Children's Nutrition Center, Little Rock, Arkansas, USA

${ }^{3}$ Department of Surgery, University of Texas Medical Branch, Metabolism Unit, Shriners Hospitals for Children, Galveston, Texas, USA

${ }^{4}$ Marlow Foods Ltd, Station Road, Stokesly, North Yorkshire, UK

Mycoprotein is a fungal-derived sustainable protein-rich food source. We have previously shown that ingesting $70 \mathrm{~g}$ mycoprotein results in a robust stimulation of muscle protein synthesis rates. We aimed to determine if a lower dose (35 g) of mycoprotein enriched with branched chain amino acids (BCAA) stimulates muscle protein synthesis rates to the same extent as $70 \mathrm{~g}$, in rested and exercised muscle of resistance-trained young men. Twenty males (age: $22 \pm 1 \mathrm{y}, \mathrm{BMI}: 25 \pm 1 \mathrm{~kg} / \mathrm{m}^{2}$ ) took part in a randomized, double-blind, parallel-group study. Participants received primed, continuous infusions of L-[ring- ${ }^{2} \mathrm{H}_{5}$ ]phenylalanine and ingested either $70 \mathrm{~g}$ mycoprotein (31.5 g protein: $2.5 \mathrm{~g}$ leucine, $1.5 \mathrm{~g}$ isoleucine, $1.9 \mathrm{~g}$ valine; MYCO; $\mathrm{n}=10$ ) or $35 \mathrm{~g}$ BCAA enriched (to match MYCO) mycoprotein (18.7 g protein: $2.5 \mathrm{~g}$ leucine, $1.5 \mathrm{~g}$ isoleucine, $1.9 \mathrm{~g}$ valine; ENR; $n=9$ ), following a bout of unilateral resistance-type exercise. Blood 
and quadriceps muscle were sampled before exercise and protein ingestion, and during a $4 \mathrm{~h}$ postprandial period to assess mixed muscle fractional protein synthetic rates (FSR). Postprandial plasma BCAA concentrations increased with protein ingestion in both groups $(\mathrm{P}<0.001)$ but peaked more rapidly and to a larger degree in ENR compared with MYCO $\left(939 \pm 37 \mu \mathrm{mol} \cdot \mathrm{L}^{-1}\right.$ at $30 \mathrm{~min}$ vs $608 \pm 28 \mu \mathrm{mol} \cdot \mathrm{L}^{-1}$ at $75 \mathrm{~min}$, respectively). Mixed muscle FSR increased following MYCO (from $0.025 \pm 0.006$ to $0.057 \pm 0.004 \% \cdot \mathrm{h}^{-1}$ in rested, and $0.024 \pm 0.007$ to $0.072 \pm 0.005 \% \cdot \mathrm{h}^{-1}$ in exercised muscle; $\mathrm{P}<0.0001$ ) and ENR (from $0.031 \pm 0.003$ to $0.043 \pm 0.005$ $\% \cdot \mathrm{h}^{-1}$ in rested, and $0.027 \pm 0.005$ to $0.052 \pm 0.005 \% \cdot \mathrm{h}^{-1}$ in exercised muscle; $\mathrm{P}<0.01)$ ingestion. FSRs increased to a greater extent following MYCO compared with ENR ingestion $(\mathrm{P}<0.05)$, and postprandial FSRs were greater in MYCO compared with ENR $(\mathrm{P}<0.01)$. We conclude that the ingestion of $35 \mathrm{~g}$ BCAA enriched mycoprotein stimulates resting and post-exercise muscle protein synthesis rates in healthy young men, but to a lesser extent compared with the ingestion of $70 \mathrm{~g}$ mycoprotein.

Equation Based on Anthropometry to Predict Body Fat Percentage by DXA in Elite Youth Soccer Athletes

A Batista ${ }^{1}$, D Granja $^{1}, \mathrm{R}$ Borrego

The purpose of this study was to develop a predict equation to calculate body fat percentage in youth soccer athletes from skinfold measurements, considering dual energy X-ray absorptiometry (DXA) values as a reference. Eighty-one males aged 15 to 26 years old $(18 \pm 2$ years; $73.0 \pm 8.2 \mathrm{~kg}$; $1.80 \pm 1.7 \mathrm{~m}$ ) from four different squads of an elite team participated in the study. The body fat mass was assessed through skinfold measurements at eight sites according to ISAK protocol, and by DXA. Correlation coefficients, bias, limits of agreement, and differences were used as validity measures, and regression analyses were used to develop soccer-specific prediction equations. Regression analysis generated an equation that includes skinfolds from three different sites (triceps, abdominal and front thigh) to calculate relative fat rate. The new equation for elite youth soccer athletes is the only validated equation that includes skinfolds across the body including lower-body sites.

\section{Sports Nutrition Knowledge of Gaelic Football Players}

M Renard ${ }^{1,2}$, A Anton-Solanas ${ }^{1}$, D Kelly ${ }^{2}$, C Ó Catháin ${ }^{2}$

${ }^{1}$ School of Sport Health and Applied Sciences, St Mary's University, Twickenham, UK

${ }^{2}$ Department of Sport and Health Sciences, Athlone Institute of Technology, Athlone, Ireland

Nutritional knowledge is a key factor for consideration when evaluating the dietary intake of athletes. Associations have been established between higher nutrition knowledge and improved quality of dietary intake. Given the negative impact poor nutrition can have on performance, further investigation into athlete's nutrition knowledge is warranted. The abridged questionnaire for sports nutrition knowledge (A-NSKQ) (Trakman et al., 2018) is a validated questionnaire which assesses both general and sports nutrition knowledge. Gaelic football is an invasion-based team sport and the second most popular sport in Ireland. Inadequacies in players dietary intake have been observed, but no previous assessment of players nutrition knowledge has been made. This study examines players knowledge using the A-NSKQ and compares results by playing level, highest level of education obtained and history of formal nutrition education. The A-NSKQ was disseminated online to a convenience sample of male Gaelic football players $(n=121$, mean age $=24 \pm 5 y$ ). This included 39 senior club players (sub-elite), 26 under 21 inter-county players (elite), and 56 senior inter-county players (elite). Total A-NSKQ score was
$42 \pm 12 \%$, classified as "poor" and lower than previous findings. Gaps in knowledge were evident and included protein recommendations, micronutrients and supplementation. There were no differences between playing level, but when grouped by highest level of education those with master's degree demonstrated higher scores by $11 \%$ in comparison to leaving certificate (upper secondary) $(\mathrm{P}<.001, \mathrm{~d}=1.17)$ and $11 \%$ in comparison to honours degree $(\mathrm{P}=.016, \mathrm{~d}=1.05)$. Those with previous formal nutrition education also demonstrated higher scores by $10 \%(\mathrm{P}<.001$, $\mathrm{d}=.93$ ). Gaelic football players would benefit from evidence-based educational interventions. Future research should consider assessment of both nutrition knowledge and dietary intake to examine any direct influence upon behaviour and subsequently sporting performance.

\section{New Equation to Estimate Body Fat Mass in International-Elite Soccer Referees}

C Petri ${ }^{1,2}$, VH Teixeira ${ }^{3}$, P Izzicupo ${ }^{4}$, G Galanti $^{5}$, A Pizzi $^{6}$, G Mascherini $^{5}$

${ }^{1}$ A.C.F. Fiorentina, Florence, Italy

${ }^{2}$ Gatorade Sports Science Institute, PepsiCo Global Nutrition R\&D, Beaumont Park, Leicester, UK

${ }^{3}$ Faculty of Nutrition and Food Sciences (FCNA), University of Porto, Rua Dr. Roberto Frias, 4200-465 Porto, Portugal

${ }^{4}$ Department of Medicine and Aging Science, “G. D'Annunzio" University of Chieti-Pescara

${ }^{5}$ Sports and Exercise Medicine Unit, Clinical and Experimental Department, University of Florence, Italy

${ }^{6}$ A.I.A., Italian Referees Association, Italy

In soccer referees, body fat mass (FM) is commonly estimated by skinfolds or dual-energy X-ray absorptiometry (DXA). The main aim was to crossvalidate the skinfolds for quantifying body fat against a recent model of DXA and to develop a specific equation for this population. This study involved a group of 43 international-level, elite male soccer referees (age $39 \pm 4$ y) to participate in World Cup in Russia 2018. Methodology used for the assessment of body composition was made with the integration of anthropometry, skinfold thickness and DXA. Anthropometric measurements were taken following ISAK protocol. Percentage of FM from skinfold thickness was then calculated from the main eight equations most commonly used: 1. Yushaz (Yuhasz 1962), 2. Faulkner (Faulkner 1968); 3. Eston et al. (Eston et al. 2005); 4. Durnin \& Womersley (Durnin and Womersley 1974); 5. Reilly et al. (Reilly et al. 2009), 6. Deuremberg et al. (Deurenberg et al. 1991), 7. Suarez et al. (Suarez-Arrones et al. 2018) and 8. the mean of all of these equations as suggested Mascherini et al. (2015). All skinfold equations (with the exception of Eston) showed large-to-very large positive correlations ( $\mathrm{r}$ from 0.71 to $0.78, \mathrm{p} \leq 0.01$ ) with DXA. All the sum of skinfolds showed moderate-to-very large positive correlations with DXA ( $\mathrm{r}$ from 0.41 to $0.76, \mathrm{p} \leq 0.01)$. The best equation found was FM\% $=8.5+(0.572 \times$ supraspinal skinfold $)+(0.252 \times$ abdominal skinfold $)$ based on significance, coefficient of determination, and sample size, included supraspinal skinfold and abdominal skinfold $\left(\mathrm{P}<.001 ; \mathrm{R}^{2} \mathrm{adj}=.589 ; \mathrm{df}=40\right)$. Almost all the equations showed positive correlations, but with different values in comparison with a recent model of DXA. Finally, the sum of skinfolds appears to be a good alternative when limited time and budget is available.

Is it Necessary to Ingest Carbohydrates and Fluids During 10 km Open Water Swimming in Top-Level Competitions?

G Olcina ${ }^{1}$, R Timón ${ }^{1}$, I González-Pérez ${ }^{2}$

${ }^{1}$ Sport Sciences Faculty, University of Extremadura, Spain

${ }^{2}$ Department of Physical and Rehabilitation Medicine and Physiotherapy, Faculty of Health Sciences, University of Alfonso X El Sabio, Spain 
Open-water swimming (OWS) is an Olympic discipline in the distance of $10 \mathrm{~km}$. Usually, competitions are based on 4 or 5 laps where in each of them swimmers have the option to stop in the feed zone to intake some food or fluids or continue swimming. The choice made may have some tactical or physiological implications. The aim of this study was to evaluate the physiological consequences of a pre-programmed race nutrition plan in toplevel open-water swimmers to know whether it can be skipped or whether it should be necessary. In three different competitions (LEN open-water cup, FINA world marathon series and FINA world championships) 22 swimmers belonging to the National Spanish Team were recruited (13 males and 9 females). PRE- and POST-race blood glucose ( $\mathrm{mg} / \mathrm{dl}$ ) and body weight $(\mathrm{kg})$ were measured. Carbohydrate $(\mathrm{CHO})$ and fluid ingestion during the race was recorded and sweat rate $(1 / \mathrm{h})$ was calculated. Blood lactate $(\mathrm{mmol} / \mathrm{L})$ was determined 5 minutes after finishing the event. T-test for comparing PRE/ POST data and bivariate Pearson correlation test for all variables were used. For an average $2 \mathrm{~h}$ duration of swimming races with a water temperature of $23 \pm 3^{\circ} \mathrm{C}$, swimmers ingested $500 \mathrm{ml}$ of fluid $+30 \mathrm{~g}$ CHO between $15^{\prime}$ before starting and 500-750 $\mathrm{ml}$ of fluid $+90 \mathrm{~g} \mathrm{CHO}$ during the race. Sweat rate was $1.09 \pm 0.46 \mathrm{~L} / \mathrm{h}$, body weight decreased after competition (PRE $71.55 \pm 7.54 \mathrm{vs}$ POST 70.90 $\pm 7.61, \mathrm{p}<0.001$ ), finish lactate was $4.1 \pm 1.9 \mathrm{mmol} / \mathrm{L}$ and blood glucose $(\mathrm{mg} / \mathrm{dl})$ increased from PRE $100 \pm 11$ to POST $120 \pm 27, \mathrm{p}<0.002$. Lactate correlated with POST glucose $(r=0.629, \mathrm{p}<0.001)$ as well as the position in overall classification correlated with POST glucose $(r=0.640, p<$ 0.001). However, in the world championships event, stronger correlations were found between overall classification and finish glucose $(\mathrm{r}=0.958, \mathrm{p}<$ $0.05)$ and between POST glucose and finish lactate $(\mathrm{r}=0.955, \mathrm{p}<0.05)$. These data suggest that fluid replacement during OWS-10 km races is not as important as $\mathrm{CHO}$ ingestion, due to the importance of $\mathrm{CHO}$ metabolism. With this information, coaches and swimmers may develop different race nutritional plans that guarantee $\mathrm{CHO}$ ingestion and do not compromise tactical actions during competition. This work has been supported by Royal Spanish Swimming Federation and the Government of Extremadura (Ref: GR18003).

\section{Day 1 Orals: Health Theme}

\section{Tuesday, 17th December 2019}

\subsection{0}

The effect of New Zealand Blackcurrant supplementation on recovery from muscle damage induced by drop jumps

R Costello, MET Willems, SD Myers, F Myers, NA Lewis, SD Blacker

\section{$\mathbf{1 4 . 1 5}$}

Comparison of selected spirometric systems for measuring the resting metabolic rate

H Bauhaus, A Vassiliadis, H Braun, HG Predel, $M$ Thevis

\subsection{0}

Longitudinal changes in body fat assessed by DEXA are adequately reflected by skinfold measurements according to ISAK standards

SL Danen, IJM Ceelen, KL Jonvik, KJM Paulussen, FC Wardenaar, LJC van Loon, JW van Dijk

\section{$\mathbf{1 4 . 4 5}$}

Carbohydrate for Endurance Athletes in Competition Questionnaire (CEAC-Q): validation of a practical and time-efficient tool for knowledge assessment

\section{G Sampson, JN Pugh, JP Morton, JL Areta}

\subsection{0}

No differences in cardiorespiratory fitness or fat oxidation between Indian and Chinese men with elevated body mass index

$M$ Demashkieh, $R$ Dalan, BO Boehm, SF Burns

\subsection{5}

Body composition in professional football players: a method comparison

NYJ Brinkmans, LJC van Loon, JW van Dijk

The Effect of New Zealand Blackcurrant Supplementation on Recovery From Muscle Damage Induced by Drop Jumps

R Costello ${ }^{1}$, MET Willems ${ }^{1}$, SD Myers ${ }^{1}$, F Myers ${ }^{2}$, NA Lewis ${ }^{3}$, SD Blacker ${ }^{1}$

${ }^{1}$ Institute of Sport, University of Chichester, Chichester, UK

${ }^{2}$ Faculty of Science, University of Portsmouth, Portsmouth, UK

${ }^{3}$ English Institute of Sport, Bath, UK

New Zealand blackcurrant (NZBC) is a rich source of anthocyanins, which improve blood flow and display anti-inflammatory and antioxidant properties that may improve recovery from exercise-induced muscle damage (EIMD). Limited evidence is available as to whether anthocyanin supplements can aid recovery in the days following muscle damaging exercise. The aim of this study was to examine if NZBC extract improves recovery following muscle damaging exercise. Following a double-blind, repeated crossover design, 12 recreationally active males (mean \pm SD: age $29 \pm 6$ years, stature $1.80 \pm 0.07 \mathrm{~m}$, body mass $78.0 \pm 10.7 \mathrm{~kg}, \Sigma$ of 4 skinfolds $35.65 \pm$

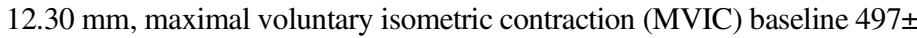
$120 \mathrm{~N}$ ) ingested either $2 \times 300 \mathrm{mg} \cdot \mathrm{day}^{-1}$ capsules with a NZBC extract (CurraNZ $^{\mathrm{TM}}$; each containing $105 \mathrm{mg}$ anthocyanin) or a visually matched placebo (PLA) 7-days prior and 3-days after completing a 100-drop jump protocol (100-DJP). Measures of MVIC, electrically stimulated (ES) contractions, countermovement jumps (CMJ), perceived muscle soreness (visual analogue scale), serum interleukin-6 (IL-6) and prostaglandin-E2 (PGE2) were made pre- (baseline), immediately-, 24-, 48- and 72 h-post the 100-DJP. MVIC, ES, CMJ and muscle soreness variables were analysed using a mixed model ANOVA with significance set at $p<0.05$. MVIC peak force was reduced immediately-post 100-DJP, compared to baseline (NZBC: $90 \pm 10$; PLA: $93 \pm 11 \% ; P=0.001, \eta p^{2}=0.320$ ), but returned to baseline at $24 \mathrm{~h}$ with no difference between groups $(P=0.940)$. ES doublet peak force was reduced compared to baseline immediately-24-, 48- and 72 h-post $(P<0.001)$ with no difference between groups $(P=0.798)$. Perceived muscle soreness increased immediately-post (NZBC: $4 \pm 2$; PLA: $\left.3 \pm 1, P=0.0001, \eta \mathrm{p}^{2}=0.417\right)$, but returned to baseline by $72 \mathrm{~h}$-post, with no difference between groups $(P=0.404)$. PGE2 declined immediately-post compared to baseline $\left(P=0.009, \eta \mathrm{p}^{2}=0.225\right)$ and returned to baseline by $72 \mathrm{~h}$ in both groups $(P=0.565)$. CMJ and IL-6 were unaffected by the 100 DJP or NZBC extract $(P>0.05)$. In conclusion, the NZBC extract did not accelerate recovery of MVIC or ES doublet peak force, perceptions of muscle soreness or inflammation following muscle damaging exercise in recreationally active males and large inter-individual variation in responses were present.

Comparison of Selected Spirometric Systems for Measuring the Resting Metabolic Rate

H Bauhaus $^{1,2}$, A Vassiliadis ${ }^{3,4}$, H Braun ${ }^{1,2}$, HG Predel $^{3}$, M Thevis ${ }^{1}$

${ }^{1}$ Institute of Biochemistry

${ }^{2}$ German Research Centre of Elite Sports

${ }^{3}$ Institute of Cardiology and Sports Medicine

${ }^{4}$ High Performance Center Rhineland

The resting metabolic rate (RMR) is predominantly known in a clinical context as an important factor for the use in bedridden patients. Recently, RMR became more relevant among athletes in the field of sports nutrition as part of the discussion on the relative energy deficiency in sport (RED-S). 
There is some evidence that the physiological RMR is lowered as a result of a low energy availability. In practice, RMR can be simply estimated using different formulas, which are based on anthropometric data (e.g. height, body mass, fat-free mass), or can be determined via indirect calorimetry measuring oxygen consumption and carbon dioxide production. To date, there is no clearly standardized procedure for the valid and reliable measurement of the RMR in the field of indirect calorimetry. Various publications, in which the RMR is examined as a criterion for a given RED-S, use different devices and different formulas. Still, due to a lack of data it is not clear to what extent these are comparable. In the present work, three spirometric systems were used to determine RMR: MetaMax 3B (Cortex, Leipzig, Germany), MasterScreen CPX (Vyaire, Hoechberg, Germany) and Q-NRG+ (Cosmed, Rome, Italy). The heart rate was measured simultaneously as well as the perception of the measurement was documented after the application of each device. 26 trained male and female subjects ( $25 \pm 3 \mathrm{y}, 10 \pm 3 \mathrm{~h} / \mathrm{wk}$ training) underwent three consecutive measurements over a single day in a randomized order. The results show a mean RMR measured by Cortex of $1870 \pm 323 \mathrm{kcal} / \mathrm{d}$, Vyaire $1829 \pm 405 \mathrm{kcal} / \mathrm{d}$ and Cosmed of $1533 \pm 309 \mathrm{kcal} / \mathrm{d}$. Cosmed shows a significant difference from Cortex and Vyaire $(\mathrm{p}<.001)$. There is a strong correlation between all devices $(p<.001)$. There were no significant results on correlations between heart rate, perception of the measurement and the RMR outcome. The current study provides further evidence that RMR can highly vary within subjects when different systems are used. Therefore, repeated RMR measurements should always be performed with the same system following the same standardized instructions.

Longitudinal Changes in Body Fat Assessed by DEXA are Adequately Reflected by Skinfold Measurements According to ISAK Standards

SL Danen $^{1}$, IJM Ceelen ${ }^{1}$, KL Jonvik ${ }^{1}$, KJM Paulussen ${ }^{1}$, FC Wardenaar ${ }^{1,3}$, LJC van Loon ${ }^{1,2}$, JW van Dijk ${ }^{1}$

${ }^{1}$ HAN University of Applied Sciences, Nijmegen, The Netherlands

${ }^{2}$ Maastricht University Medical Centre+, Maastricht, The Netherlands

${ }^{3}$ Arizona State University, Phoenix, Arizona, USA

Dual-energy X-ray absorptiometry (DEXA) is widely recognized as a reliable method for the assessment of body fat in athletes. It is unclear whether the more practical assessment of skinfold thickness adequately reflects changes in body fat over time. In the current study we assessed whether changes in skinfold thickness are associated with changes in body fat as assessed by DEXA. This observational cohort study included fifty-seven recreationally active males (age: $27 \pm 6 \mathrm{y}$; BMI: $24 \pm 3 \mathrm{~kg} \cdot \mathrm{m}^{-2}$ ). DEXA and 8-site skinfold measurements according to International Society for the Advancement of Kinanthropometry (ISAK) standards were performed under strictly standardized conditions at baseline and after twelve weeks of endurance exercise training. Associations between changes in the sum of 8 skinfolds and body fat and were assessed by Pearson correlation. At baseline, the sum of 8 skinfolds $(108.1 \pm 37.3 \mathrm{~mm})$ correlated very strongly with fat mass $(18.1 \pm 5.5 \mathrm{~kg} ; \mathrm{r}=0.87, P<0.001)$ and body fat percentage $(22.6 \pm 5.1 ; \mathrm{r}=0.89, P<0.001)$ derived from DEXA. Over the 12-week period, the change in the sum of 8 skinfolds $(-1.4 \mathrm{~mm}$, [95\% CI -4.5 to 1.8$])$ correlated strongly $(\mathrm{r}=0.73, P<0.001)$ with the change in body fat mass $(-0.5 \mathrm{~kg}[95 \% \mathrm{CI}-0.9$ to -0.2$])$. In line, the change in the sum of 8 skinfolds correlated strongly $(\mathrm{r}=0.69, P<$ 0.001) with the change in body fat percentage ( $-0.6 \%$ [95\% CI -0.9 to $-0.2]$ ). We conclude that small changes in body fat as assessed by DEXA are adequately reflected by changes in the sum of 8 skinfolds. Hence, skinfold measurements according to ISAK standards can be considered an accurate and practical tool for monitoring changes in body fat in fieldbased settings.
Carbohydrate for Endurance Athletes in Competition Questionnaire (CEAC-Q): Validation of a Practical and Time-Efficient Tool for Knowledge Assessment

G Sampson, JN Pugh, JP Morton, JL Areta

School of Sport and Exercise Sciences, Liverpool John Moores University, UK

Carbohydrate intake is key for endurance performance, but there is currently no practical and time-efficient tool available to assess athletes' knowledge gaps of carbohydrate for competition. This study validated a new practical multiple-choice questionnaire designed to quickly determine endurance athletes core knowledge of carbohydrate intake to maximise endurance performance (CEAC-Q). The questionnaire was developed by a group of experts based on the most current scientific evidence. It consisted of 25 questions divided into 5 sub-sections, namely carbohydrate metabolism, loading, pre-race meal, during race and recovery. The questionnaire was delivered online with each sub-section worth 20 points and a total possible score of 100 . A between-groups analysis of variance compared scores in three different population groups to assess construct validity: general population (GenP; $n=67)$, endurance athletes $(E A ; n=145)$, and registered sports nutrition practitioners (SNP; $n=60)$. A significant difference in total nutrition scores $($ mean \pm SD) was observed between all pairwise comparisons; GenP $(17 \pm 20 \%)$, EA $(46 \pm 195 \%)$ and SNP $(76 \pm 10 \%)(\mathrm{p}=<0.001)$. For each sub-section, scores were also significantly different between groups with mean scores of $3.4 \pm 4.7 \%$ (GenP), 9.2 $\pm 5.2 \%$ (EA); $15.2 \pm 3.5 \%$ (SNP) $(\mathrm{p}=0.001)$. Athletes working with a SNP $(\mathrm{n}=24,17 \%)$ had higher knowledge scores $(56 \pm 25 \%)$ than those who were self-taught $(\mathrm{n}=83,45 \pm 18 \%, \mathrm{P}=0.025)$. Retest of the CEAC-Q 10-14 days after, in a subgroup of $59 \mathrm{EA}$ resulted in an increase of test score in 54 of them (92\%), with a mean increase in score of $8 \pm 14 \%$ points $(\mathrm{p}=<0.001)$. The CEAC-Q is a psychometrically valid new practical and time-efficient tool to assess athletes' knowledge of carbohydrate for competition in less than 10 and 5 minutes for athletes and practitioners, respectively, allowing for quick and accurate identification and implementation of strategies to optimise nutrition for performance.

\section{No Differences in Cardiorespiratory Fitness or Fat Oxidation Between Indian and Chinese Men With Elevated Body Mass Index}

M Demashkieh $^{1}$, R Dalan ${ }^{2,3}$, BO Boehm ${ }^{2,3}$, SF Burns ${ }^{1}$

${ }^{1}$ Physical Education and Sports Science, National Institute of Education, Nanyang Technological University, Singapore

${ }^{2}$ Lee Kong Chian School of Medicine, Nanyang Technological University, Singapore

${ }^{3}$ Department of Endocrinology, Tan Tock Seng Hospital, National Healthcare Group, Singapore

There is a higher prevalence of type 2 diabetes mellitus (T2DM) in Indians than Chinese in Singapore. Previous data have demonstrated that Indians have lower cardiorespiratory fitness and reduced fat oxidation during exercise than Europeans which are associated with lower insulin sensitivity. This cross-sectional study examines if similar differences in cardiorespiratory fitness and fat oxidation exist between Chinese and Indian men with elevated body mass index (BMI) $\left(23.5-30.0 \mathrm{~kg} / \mathrm{m}^{2}\right)$. Four Indian [mean (SD): aged 30 (2) y] and nine Chinese [mean (SD): aged 31 (7) y] sedentary men consented to visit the laboratory on two occasions after an overnight fast. On their first visit, participants completed a submaximal walking test, to determine their predicted maximal oxygen uptake $\left(\mathrm{VO}_{2} \mathrm{max}\right)$. For their second visit, resting metabolic rate was measured for 30 minutes before participants completed a walking test to determine their fat oxidation during submaximal exercise. Indians had a higher BMI 
than Chinese [27.6 (1.3) vs $24.7(1.8) \mathrm{kg} / \mathrm{m}^{2}, p=.012$, respectively] but despite presenting with an $6 \%$ higher waist circumference $[89.5(6.0) \mathrm{cm}$ vs $84.5(5.9) \mathrm{cm}, p=.185), 22.1 \%$ greater body fat percentage [32.6 (4.2)\% vs $26.7(6.7) \%, p=.141]$ and $3 \%$ lower fasting blood glucose [5.4 $(0.2) \mathrm{mmol} / \mathrm{L}$ vs $5.6(0.3) \mathrm{mmol} / \mathrm{L} \mathrm{p}=.344]$, these were not significantly different. Predicted $\mathrm{VO}_{2}$ max was $13 \%$ lower in Indians than Chinese [32.0 (9.0) $\mathrm{mL} / \mathrm{kg} / \mathrm{min}$ vs $36.9(5.4) \mathrm{mL} / \mathrm{kg} / \mathrm{min}$ ] but not significantly different $(p=.241)$, even after accounting for differences in BMI $(\mathrm{p}=.689)$. After controlling for BMI, fat oxidation compared at 20,22 and $24 \mathrm{ml} / \mathrm{kg} / \mathrm{min}$ did not differ between groups (all $p>.05$ ) and was also similar when comparing Indians and Chinese at the same relative exercise intensities between $40 \%$ and $55 \%$ of $\mathrm{VO}_{2} \max \left[40 \% \mathrm{VO}_{2} \max : 4.2(0.9) \mathrm{mg} / \mathrm{kg} / \mathrm{min}\right.$ vs $4.5(1.5) \mathrm{mg} / \mathrm{kg} / \mathrm{min}, p=.712 ; 45 \% \mathrm{VO}_{2} \max : 4.1(0.9) \mathrm{mg} / \mathrm{kg} / \mathrm{min}$ vs 4.1 (2.3) $\mathrm{mg} / \mathrm{kg} / \mathrm{min}, p=.964 ; 50 \% \mathrm{VO}_{2} \max : 2.9(2.1) \mathrm{mg} / \mathrm{kg} / \mathrm{min}$ vs 3.7 (2.1) $\mathrm{mg} / \mathrm{kg} / \mathrm{min}, p=.549$; $55 \% \mathrm{VO}_{2} \max : 2.9(2.0) \mathrm{mg} / \mathrm{kg} / \mathrm{min}$ vs 3.2 (2.2) $\mathrm{mg} / \mathrm{kg} / \mathrm{min}, p=.811]$. Differences in cardiorespiratory fitness and the capacity of skeletal muscle to oxidise fat may not account for differences in T2DM risk between Indian and Chinese men, although the study is ongoing and presently limited by sample size.

\section{Body Composition in Professional Football Players: A Method Comparison}

NYJ Brinkmans ${ }^{1}$, LJC van Loon ${ }^{1,2}$, JW van Dijk ${ }^{1}$

${ }^{1}$ Institute of Sports and Exercise Studies, HAN University of Applied Sciences, Nijmegen, The Netherlands

${ }^{2}$ NUTRIM School of Nutrition and Translational Research in Metabolism, Maastricht University Medical Centre, Maastricht, The Netherlands

Body composition is routinely monitored in most professional football players. Dual-energy X-ray absorptiometry (DXA) is generally accepted as a reliable method to monitor body composition, although consensus on the preferred analysis algorithm is absent. Furthermore, DXA is expensive and often not feasible for regular monitoring. Standardised skinfold measurements may be a valuable instrument for regular monitoring of body composition, although information on the agreement between skinfold measurements and DXA is lacking. Therefore, we assessed the association and agreement between body fat percentage derived from DXA, deuterium dilution (DD) and skinfold thickness. Fifty-eight professional male football players from 3 first team squads playing in the Dutch Eredivisie were included. Fat mass (FM) and fat free mass (FFM) were assessed by a whole-body DXA scan and analysed with the classic $\left(\mathrm{DXA}_{\mathrm{C}}\right)$ and NHANES $\left(\mathrm{DXA}_{\mathrm{N}}\right)$ algorithm. Fat mass was also derived from total body water as assessed by DD $(n=45)$. Skinfold thickness ( 8 sites) was assessed according to ISAK standards and translated to body fat \% (BF\%) by Juhasz and Durnin \& Womersly (D\&W) equations. Associations between methods were analysed by Pearson's correlation coefficient. Bland-Altman plots were made to assess the agreement between different methods. Mean $\mathrm{BF} \%$ by $\mathrm{DXA}_{\mathrm{C}}, \mathrm{DXA}_{\mathrm{N}}$, DD, Juhasz and $\mathrm{D} \& \mathrm{~W}$ were respectively $11.2 \pm 1.9,15.7 \pm 1.8,11.7 \pm 2.4,7.0 \pm 1.0$ and $11.2 \pm 2.5 \%$. The sum of 8 skinfolds (SF) was $54.8 \pm 13.1 \mathrm{~mm}$. Despite the relatively small data range, $\mathrm{DXA}_{\mathrm{N}}$, DD, Juhasz, D\&W and sum of $8 \mathrm{SF}$ correlated strongly with $\mathrm{BF} \%$ derived from $\mathrm{DXA}_{\mathrm{C}}(\mathrm{r}=1.00 ; \mathrm{r}=0.85 ; \mathrm{r}=79 ; \mathrm{r}=72 ; \mathrm{r}=0.81$, respectively). Despite the perfect correlation, $\mathrm{BF} \%$ derived from $\mathrm{DXA}_{\mathrm{N}}$ was systematically higher $(+4.6 \pm 0.1 \%)$ than $\mathrm{DXA}_{\mathrm{C}}$, whereas DD showed an acceptable level of agreement with $\mathrm{DXA}_{C}(+0.7 \pm 1.3 \%)$. Large proportional bias in $\mathrm{BF} \%$ was observed with the Juhasz equation when compared with $\mathrm{DXA}_{\mathrm{C}}$. In conclusion, the high level of agreement between DD and DXA $_{C}$ supports the use of the DXA $A_{C}$ analysis algorithm for professional football players. Furthermore, our data suggest that the sum of $8 \mathrm{SF}$ is the most appropriate skinfold-based approach to monitor body fat in professional football players.

\section{Day 2 Orals: Health Theme}

\section{Wednesday 18th December 2019}

\subsection{0}

Assessing adequacy of protein feeding in resistance-trained athletes; re-visited through peri-training nutrition (PTN)

M Kozior, PM Jakeman, RW Davies, C Norton

14.15

Effects of pre-sleep animal vs. plant-based protein consumption on inflammation and muscle recovery following damaging exercise

PG Saracino, HE Saylor, BR Hanna, MJ Ormsbee

14.30

The relationship between blood glucose level and race pace in trail running race

K Ishihara, N Uchiyama, S Kizaki, E Mori, A Tani, Y Nakamura

Assessing Adequacy of Protein Feeding in Resistance-Trained Athletes; Re-Visited Through Peri-Training Nutrition (PTN)

M Kozior ${ }^{1,2}$, PM Jakeman ${ }^{1,2,3}$, RW Davies ${ }^{1,2}$, C Norton ${ }^{1,2,3}$

${ }^{1}$ Department of Physical Education and Sport Sciences, University of Limerick, V94 T9PX Limerick, Ireland

${ }^{2}$ Food for Health Ireland (FHI), Centre for Interventions in Infection, Inflammation and Immunity, University of Limerick, V94 T9PX Limerick, Ireland

${ }^{3}$ Health Research Institute, University of Limerick, V94 T9PX Limerick, Ireland

Key opinion leaders in sport and exercise nutrition emphasise the importance of a personalised and periodised approach for athletic populations. The PTN paradigm proposed here places aims and characteristics of training and competition at the core of a dietary assessment, providing potential to apply this recommended approach, and identifying theretofore - unexploited opportunities for nutrient optimisation to support training adaptations. PTN acknowledges the importance of the nutrient intakes (type, quality (source), quantity, timing, distribution and frequency) to support individual training sessions and competitive demands. Our objective was to determine 1) the adequacy of reported protein (PRO) intake and 2) frequency and distribution of eating occasions (EO) relative to the demands of a resistance training (RT). Data are presented for RT athletes as Mdn (IQR) $(\mathrm{n}=32$, aged (y) $25.1(9.5)$, body mass (kg) 81.4 (14.3), a RT history $\geq 6$ months and completing 3 (3) RT sessions weekly). Dietary data was collected by self- reported 7-day weighed intake record, and analysed using Nutritics V5.09. The adequacy of PRO intake was assessed against recommended daily (1.6$\left.2.2 \mathrm{~g} \cdot \mathrm{kg}^{-1} \cdot \mathrm{d}_{-1}\right)$ and per EO ( $\left.\geq 0.25 \mathrm{~g} \cdot \mathrm{kg}_{-1}\right)$ intakes for this population. Investigation of $\mathrm{EO}$ frequency and distribution against recommendations (3-4 EO.d $\mathrm{d}_{-1}$ and distribution of every 3-5 h) was also completed. PRO feeding in support of the adaptive outcome of RT was assessed in the $24 \mathrm{~h}$ recovery phase. The reference quantity for daily PRO intake was met for $18 \%$, exceeded $\left(>2.2 \mathrm{~g} \cdot \mathrm{kg}^{-1} \cdot 24 \mathrm{~h}^{-1}\right)$ for $52 \%$ of all $24 \mathrm{~h}$ post-RT phases $\left(\mathrm{n}=61,2.2(1.3) \mathrm{g} \cdot \mathrm{kg}^{-1} \cdot 24 \mathrm{~h}^{-1}\right)$. Adequacy in PRO consumption per EO was achieved for $63 \%$ of all EO $\left(0.3(0.4) \mathrm{g} \cdot \mathrm{kg}_{-1}\right)$. The number of EO within a $24 \mathrm{~h}$ post-RT phase was 5 (3). $98 \%$ of RT sessions were supported with at least 3 EO. EO were consumed every 2 h $20 \mathrm{~min}$ ( $2 \mathrm{~h} 00 \mathrm{~min}$ ), but $26 \%$ of them were distributed every $3-5 \mathrm{~h}$. This data highlights the need for advancements in dietary assessment in athletic populations towards periodised, personalised nutrition, with training at the core of this process. PTN assessment allows for effective monitoring of habitual nutritional practices of athletes. This approach to dietary 
assessment in athletic populations could provide opportunities to further support training adaptations and confer competitive advantage.

\section{Effects of Pre-Sleep Animal vs. Plant-Based Protein Consumption on Inflammation and Muscle Recovery Following Damaging Exercise}

PG Saracino, HE Saylor, BR Hanna, MJ Ormsbee

Institute of Sports Sciences and Medicine, Florida State University, USA

The purpose of this study was to examine the effects of animal vs. plantbased pre-sleep protein on muscle recovery and inflammation following a morning bout of damaging exercise. Twenty-seven middle aged (40-64 y) men performed a morning bout of 5 sets of 15 repetitions of eccentric only knee extension and flexion (ECC) on both limbs. Participants were randomly assigned to consume $40 \mathrm{~g}$ whey hydrolysate $(\mathrm{WH})$, whey isolate (WI), rice and pea combination (RP), or placebo (PL) $30 \mathrm{~min}$ pre-sleep the night of ECC and the following two nights (24 and $48 \mathrm{~h}$ post-ECC). Participants consumed $15 \%$ total intake from protein in a standardized diet (55\% CHO, 30\% Fat) two days pre-ECC and for three days of testing. Maximal isometric voluntary contraction of the knee extensors (ISOMext) and flexors (ISOMflex) was performed pre, post, 24, 48, and $72 \mathrm{hrs}$ post-ECC. Blood samples were collected pre, post, 4, 6, 24 , 48, and $72 \mathrm{~h}$ post-ECC. Muscle soreness was rated at pre, 24, 48 and $72 \mathrm{~h}$ post-ECC. Data are presented as mean \pm SE. No differences were seen between groups at baseline for peak torque of ISOMext (WH: 182 \pm 8 , WI: $183 \pm 23$, RP: $193 \pm 14$, PL: $196 \pm 15 \mathrm{~N}-\mathrm{m} ; \mathrm{p}=0.867$ ) or ISOMflex (WH: 92 \pm 5 , WI: $86 \pm 8$, RP: $95 \pm 11$, PL: $100 \pm 6 \mathrm{~N}-\mathrm{m}$; $\mathrm{p}=0.675$ ). Significant peak torque reductions were measured for ISOMext and ISOMflex ( $\mathrm{p}<$ 0.001) post-ECC for all groups. Peak ISOMext was reduced from baseline post-ECC $(-34 \pm 7 \mathrm{~N}-\mathrm{m}, \mathrm{p}=0.001)$ and returned to baseline at $72 \mathrm{~h}$. Peak ISOMflex was reduced from baseline at 48 -h post-ECC $(-32 \pm 4$ $\mathrm{N}-\mathrm{m}$ ) and was still reduced $72 \mathrm{~h}$ post-ECC. There were no differences between groups for muscle function at any time point. Muscle soreness was elevated post-ECC $(\mathrm{p}<0.001)$, peaked at $48 \mathrm{~h}$, and remained elevated during the $72 \mathrm{~h}$ recovery period with no differences between groups. IL-6 increased following ECC ( $\mathrm{p}<0.001 ; \mathrm{n}=23)$. Plasma IL-6 concentrations were elevated at $6 \mathrm{~h}(+1.7 \pm 0.3 \mathrm{pg} / \mathrm{mL} ; 109 \%$; $<<0.001)$, returned to baseline, and increased again at $72 \mathrm{~h}(+1.3 \pm 0.3 ; 86 \% ; \mathrm{pg} / \mathrm{mL}$; $\mathrm{p}=0.014)$ compared to baseline with no group differences. When damaging eccentric exercise is performed in the morning and daily protein intake is sufficient $(1.1 \pm 0.0 \mathrm{~g} / \mathrm{kg})$, animal- or plant-based presleep protein do not appear to improve muscle recovery more than PL in middle aged men.

\section{The Relationship Between Blood Glucose Level and Race Pace in Trail Running Race}

\section{K Ishihara, N Uchiyama, S Kizaki, E Mori, A Tani, Y Nakamura}

Dept Food Sci Human Nutr, Ryukoku Univ, Kyoto, Japan

Carbohydrate intake affects endurance exercise performance. However, it is difficult to observe the relationship between blood glucose levels and race pace in the ultramarathon race, as various factors affect race pace, such as running surface and gradient as well as hydration and gastrointestinal symptom. Recently, some trail runners intend to maintain their blood glucose at lower level to maximize fat oxidation. The current study intended to measure blood glucose level during a $160-\mathrm{km}$ race using a continuous glucose-monitoring system (CGMS) and compared athlete's race pace their glucose level in real time. Seven trail runners (3 male and 4 female) including 2 national elite level runners took part in the study. A CGMS glucose sensor was attached on the back of the upper arm and the glucose profile was monitored continuously until the end of the race. The race course was divided into 11 section by timing gates. Race pace was calculated from official net time between each timing gate and was standardized to the average net time of top 30 runners (1.2\% of total 2450 race participants). Food and drink intake during the race were recorded and carbohydrate and energy intake were calculated. Overall race time was between $22.5 \mathrm{~h}$ and $29.18 \mathrm{~h}$. Observed glucose levels were varied between 3.4 to $13.9 \mathrm{mM}$ and were within normoglycemic $(4.0-13.9 \mathrm{mM})$ except for 1 value $(3.4 \mathrm{mM})$ in one subject. Average glucose level during overall race was from $5.8 \pm 0.4$ to $8.9 \pm 0.9 \mathrm{SD}$ $\mathrm{mM}$. The total amount of carbohydrate intake during the race was 427 to $1387 \mathrm{~g}$. Average glucose levels and race time between each timing gate were classified into two groups (higher and lower) by their own median value of glucose level and race pace, respectively. There was a significant relationship when comparing classified average glucose level and race pace in $2 \times 2$ contingency Pearson's kai square $(\mathrm{p}<0.05)$. In conclusion, there was a weak positive relationship between average glucose level and standardized race pace even when glucose levels were within normal range in ultra-trail race. This work was supported by a grant from Nagatasangyo Col., Ltd.

\section{Day 1 Posters}

\section{Tuesday 17th December 2019}

1. Resumption of eumenorrhea after prolonged menstrual dysfunction in an elite road-cyclist following increase in body weight in parallel with high training load: a 5-year case report

JL Areta

2. Dietary selenium intakes and their association with muscle strength and function in $\geq 85$ year old adults: the Newcastle $85+$ Study G Perri, T Hill, J Mathers, $N$ Mendonça

3. Treatment strategies to reverse bone loss in athletes with functional hypothalamic amenorrhea

\section{J Hamer, J Roche}

4. Metabolic enzyme adaptations to long-term feeding of ketogenic diet containing medium-chain triglyceride in rats

A Fukazawa, T Karasawa, Y Yokota, S Kondo, S Terada

5. Energy availability estimated in free-living conditions positively correlated with peak progesterone concentration in the menstrual cycle of race walkers and runners not using hormonal contraception M. Carolina Castellanos-Mendoza

6. Changes in the thigh and arm muscle glycogen contents in Japanese male gymnasts during normal practice

A Kamei, H Hasegawa, H Takahashi

7. The effect of an eight-week high $n-3$ and low $n-6$ PUFA dietary intervention on submaximal and peak oxygen consumption in trained cyclists.

L. J. Hale., G. Davison., L. Passfield., B. Gyimah., M. R. Lindley

8. The effect of an endurance exercise bout on substrate utilisation at increasing intensities: characterising the $\mathrm{FAT}_{\max }$ curve after exercise with and without carbohydrate ingestion

J Bartley, S Barrows, H Strickler, C Savage, JL Areta

9. Assessing body fat percentage in professional jockeys using DXA and skinfold prediction equations

A Dunne, GD Warrington, S O'Connor A McGoldrick, J Pugh, $M$ Harrison, $G$ O'Loughlin, SJ Cullen

10. Magnesium status in elite track \& field athletes: an 8-year analysis of the British Athletics World Class Performance team SC Killer, $R$ Chakraverty, I Taylor, $N$ Pollock

11. Do triathletes follow a proper nutritional strategy during Olympic distance triathlon?

$G$ Olcina, C Crespo, A Camacho-Cardeñosa, M CamachoCardeñosa, I Martinez-Guardado, R Timón

12. Using behaviour science to develop a sport nutrition behavioural assessment tool

MRN Bentley, L Patterson, N Mitchell, SH Backhouse 
Resumption of Eumenorrhea After Prolonged Menstrual Dysfunction in an Elite Road-Cyclist Following Increase in Body Weight in Parallel With High Training Load: A 5-Year Case Report

JL Areta

\section{Liverpool John Moores University, Liverpool, UK}

Chronic low energy availability typically results in menstrual dysfunction and/or low bone mineral density. It is unclear how this condition, known as the female athlete triad, affects physical capacity and what the recovery time-course of menses is with increased energy availability concomitant to high training load. In this case-study, an amenorrheic elite female cyclist $\left(\mathrm{VO}_{2 \max } 3.54 \mathrm{~L} / \mathrm{min}, 64 \mathrm{ml} / \mathrm{min} / \mathrm{kg}\right.$, PPO $300 \mathrm{~W}, 5.4 \mathrm{~W} / \mathrm{kg}$ and 23 years in 2014) is shown to resume normal menstrual function after a prolonged period of menstrual dysfunction (amenorrhea (2013-2015)/oligomenorrhea (2015-2018)) while engaging in high training load and in competition. Resumption of menses was recorded in June 2018, 5-6 months after a weight gain of $\sim 10 \%$ ( $\sim 5 \mathrm{~kg}$ ) displaying regular menses (every $23-35$ days) ever since. Training load and volume increased from 2014 to 2019 (26707 to $41945 \mathrm{TSS} /$ year and 584 to $818 \mathrm{~h} /$ year, respectively). During the period of menstrual dysfunction weight was $51.3 \pm 2.3 \mathrm{~kg}$ (mean $\pm 95 \% \mathrm{CL} ; 80$ records), and fat percentage 19\% (DXA, 2016), and after an episode of weight gain $56.8 \pm 2.6$ (35 records) $(\mathrm{p}<0.001)$ and fat percentage $25 \%$ (DXA, 2019). Crank-based power-meter data was recorded for training sessions and competitions, and mean maximal power (MMP) over the range of 5 seconds to $4 \mathrm{~h}$ improvements in absolute power (watts) through the 2014-2019 period, while relative MMP (watts/kg) likely peaked in the 2015-16 season for 5', 20' and 30' and remained mostly unaffected across seasons despite the increase in body mass. The athlete achieved podium placings consistently in national and international competitions and achieved best performances in the 2018-2019 season. These findings suggest that 1) best relative power output of 5 ' $-1 \mathrm{~h}$ seemed to be achieved despite low energy availability and menstrual dysfunction, 2) high performance can be achieved at an elite level despite an increase in body weight in a weight-sensitive sport and 3) despite high training loads when coupled with high energy availability.

Dietary Selenium Intakes and Their Association With Muscle Strength and Function in $\geq 85$ Year Old Adults: The Newcastle 85+ Study

G Perri $^{1}$, T Hill ${ }^{1}$, J Mathers ${ }^{1}$, N Mendonça ${ }^{2}$

${ }^{1}$ Institute of Cellular Medicine, University of Newcastle, UK

${ }^{2}$ Marie Skłodowska Curie Research Fellow, University of Lisbon, Portugal

Selenium is an essential micronutrient with biochemical and cellular effects through activities of 25 selenocysteine-containing selenoproteins. Selenoproteins are anti-inflammatory and have antioxidant properties. Severe selenium deficiency causes muscle weakness and atrophy in humans but the effects of moderate selenium deficiency are unclear. The aims of this study are: to determine dietary selenium intakes and contributing food sources in very old adults and; to determine whether selenium intakes are associated with 5-year trajectories of muscle function: hand-grip strength (HGS) and Timed-Up-and-Go (TUG). Cross-sectional (baseline) and prospective (1.5, 3 and 5-year follow-up) analyses of 845 participants aged 85 years from the Newcastle 85+ study were assessed for HGS and TUG using standardized protocols (Antoneta et al. 2016). Baseline dietary intakes were assessed using 24-hour multiple pass recall methods on two separate days (Mendonça et al. 2016). The top selenium food contributors $(\sim 90 \%)$ and the adequacy of intakes were determined i.e. those with intakes < LRNI, between the LRNI and RNI and > RNI. Linear mixed models explored associations between selenium intake categories and time on prospective, 5-year changes in HGS and TUG in all participants, males and females. Median intakes of selenium were 39,48 and $35 \mu \mathrm{g}$, respectively. Selenium intakes were below the LRNI in $51 \%$ of participants (median $27 \mu \mathrm{g}$ ) whilst $15 \%$ had intakes $\geq$ the RNI (median $85 \mu \mathrm{g}$ ). Only $13.3 \%$ of females and $16.9 \%$ of males met the RNI. The top selenium contributors were cereals (46\%), meat (22\%), fish (10\%), milk (6\%), eggs $(4 \%)$ and potatoes $(3 \%)$ making up $91 \%$ of selenium intakes. Those with the lowest intakes had $2.72 \mathrm{~kg}$ lower HGS and 2.36s slower TUG compared to those with higher intakes $(\mathrm{P}<0.005)$. There was no association between selenium intake in HGS or TUG, but time had a significant effect on the rate of change over 5 -years in both parameters $(\mathrm{P}<0.001)$. Overall these results show that poor dietary selenium intakes are common in very old adults and that cereal and cereal products are major sources of selenium in this population. Whilst low selenium intakes are associated with worse HGS and TUG performance in cross-sectional analyses, no significant associations were observed in the prospective analyses.

\section{Treatment Strategies to Reverse Bone Loss in Athletes With Func-} tional Hypothalamic Amenorrhea

J Hamer, J Roche

University College London, London, UK

Functional hypothalamic amenorrhea (FHA) is characterized by the loss of the menstrual cycle due to the suppression of the hypothalamic pituitary ovarian axis (HPOA). FHA in athletes can be a symptom of relative energy deficiency in sport (RED-S). RED-S has expanded the concept of the Female athlete triad acknowledging that this syndrome affects many aspects physiological function including metabolic rate, menstrual function, bone health, immunity, protein synthesis, cardiovascular function and psychological health. The fundamental issue with RED-S is a mismatch between energy availability (EA) (the amount of dietary energy remaining after exercise training for all physiological functions each day) and energy expenditure (EE). It is well established that the endocrine disruptions and a state of low EA directly impair bone health through the suppression of the key metabolic hormones required for bone formation (leptin, IGF-1 and T3) and the hypoestrogenic environment upregulating osteoclastic bone resorption. This leads to a loss in bone mineral density (BMD), thus increasing the risk of stress fractures and osteoporosis. To support the continued development of clinical guidelines in treating athletes with FHA, a systematic review of the literature was conducted to document the current evidence regarding the efficacy of treatment strategies to address bone loss in athletes with FHA. The primary treatment of FHA in female athletes is the modification of diet and exercise behaviours to enable an increase in EA and weight restoration if required. Restoring energy availability and the return of menses are essential to enable athletes with FHA to reverse bone loss, and this should not be understated. If an athlete experiences further health complication, whilst restoring EA (e.g. stress fractures or if menstruation is not restored after an adequate trial of restoring EA), transdermal oestradiol and cyclic micro-ionized progesterone may be prescribed. This will be used as a short-term treatment to protect bone health until the underlying issue of low energy availability is resolved. Ackerman et al (2018) concluded the oral contraceptive pill should not be prescribed to these athletes due to the hepatic first pass effects on the liver leading to downregulation of insulin growth factor-1, further exacerbating bone loss.

\section{Metabolic Enzyme Adaptations to Long-Term Feeding of Ketogenic Diet Containing Medium-Chain Triglyceride in Rats}

A Fukazawa, T Karasawa, Y Yokota, S Kondo, S Terada

Department of Life Sciences, Graduate School of Arts and Sciences, The University of Tokyo, Tokyo, Japan

Very low-carbohydrate, high-fat (ketogenic) diets are recently receiving much attention from athletes, because long-term intake of the diet enhances 
production and utilization of ketone bodies, which are more energyefficient fuels for skeletal muscle. However, adaptation to the extremely low-carbohydrate diet has been shown to upregulate pyruvate dehydrogenase kinase 4 (PDK4) content in skeletal muscle, which is a negative regulator of glycolytic flux, resulting in the impaired high-intensity exercise capacity. Because medium-chain triglyceride (MCT) is capable of producing more ketone bodies than long-chain triglyceride (LCT), incorporating MCT into the diet may allow more carbohydrates yet preserving ketosis and exert less inhibitory effect on muscle glucose metabolism. The purpose of this study was therefore to examine the effects of long-term feeding of ketogenic diet containing MCT on key metabolic enzymes in rat skeletal muscle. Male Wistar rats (9-week-old) were placed on a standard diet (CON group, PFC ratio $=21: 16: 63$ ), ketogenic diet containing LCT (LKD group, PFC ratio $=12: 87: 1$ ) or MCT (MKD group, PFC ratio = 16:66:18) for $24 \mathrm{wks}$. Despite the lower lipid content in the diet, plasma $\beta$ hydroxybutyrate concentration in the MKD group increased to a level similar to that attained in the LKD group. In soleus and plantaris muscle, protein content of a ketolytic enzyme, 3-oxoacid CoA transferase 1 in the MKD group, not the LKD group, was significantly higher than that observed in the CON group. Whereas the LKD group had about 3-fold higher PDK4 protein content in skeletal muscle, such increase in PDK4 was not observed in the MKD group. In addition, muscle glycogen content in the LKD group, not the MKD group, was significantly lower compared with that in the CON group. In conclusion, long-term intake of ketogenic diet containing MCT may enhance ketone bodies utilization capacity in skeletal muscle without exerting inhibitory effects on glucose metabolism.

Energy Availability Estimated in Free-Living Conditions Positively Correlates With Peak Progesterone Concentration in the Menstrual Cycle of Race Walkers and Runners not Using Hormonal Contraception

\section{Carolina Castellanos-Mendoza}

Faculty of Health Sciences and Sport, University of Stirling, United Kingdom

Low energy availability [EA] alters luteinizing hormone [LH] and oestradiol, important menstrual cycle $[\mathrm{MC}]$ regulators. The aim was to determine the relationship between EA estimated in free-living conditions and peak serum progesterone concentration [Pk-PRG], as the ovulatory status indicator of MCs. Subjects were 8 race walkers and 9 runners of recreational-world competitive level, $47-109 \mathrm{~km} \cdot \mathrm{week}^{-1}$ training volume, 14-41 years old, 1.5-27 years after menarche, 39.5-61.5 kg body mass, 1.39-1.74 m height, and 71-162 mm $\sum 8$ skinfolds [methodology of the International Society for the Advancement of Kinanthropometry]. Twentyone MCs were analysed. Seven-day average EA was estimated, within the follicular phase of each MC, from food and training \& physical activity logs. Individual urine LH-peak day, predicted MC length and basal body temperature changes were considered to schedule progesterone determination at peak period. Serum progesterone was determined by electrochemiluminescence immunoassay with Cobas e601. EA correlates with Pk-PRG $[r(19)=.72,95 \%$ CI: .41-.88; $p<.001 ; 1-\beta=.99]$. EA of cycles with optimal $\left[>10 \mathrm{ng} \cdot \mathrm{mL}^{-1}\right]$ was higher than EA of cycles with anovulatory $\left[<5.8 \mathrm{ng} \cdot \mathrm{mL}^{-1}\right] \mathrm{Pk}-\mathrm{PRG}$ [one-way ANOVA, $1-\beta=.84$; Tukey's $p=.008]: 40.0 \pm 4.5[\mathrm{n}=8]$ vs. $30.3 \pm 2.9[\mathrm{n}=4] \mathrm{Kcal} \cdot \mathrm{Kg}$ $\mathrm{FFM}^{-1} \cdot$ day $^{-1}$. Ovulatory disturbances $\left[\mathrm{Pk}-\mathrm{PRG} \leq 10 \mathrm{ng} \cdot \mathrm{mL}^{-1}\right.$ ] were observed with EA $\leq 36 \mathrm{kcal} \cdot \mathrm{Kg} \mathrm{FFM}^{-1} \cdot \mathrm{day}^{-1}$. Female athletes not using hormonal contraception should monitor the ovulatory status of their MCs, otherwise aim for EA $>36 \mathrm{kcal} \cdot \mathrm{Kg} \mathrm{FFM}^{-1} \cdot \mathrm{day}^{-1}$-estimated with methods used herein.
Keywords: female athletes, ovulation, anovulation

\section{Changes in the Thigh and Arm Muscle Glycogen Contents in Japanese} Male Gymnasts During Normal Practice

\author{
A Kamei ${ }^{1}, \mathrm{H}_{\text {Hasegawa }}{ }^{2}, \mathrm{H}$ Takahashi ${ }^{1}$ \\ ${ }^{1}$ Japan Institute of Sports Sciences, Japan \\ ${ }^{2}$ Matsumoto University, Japan
}

To systematically examine the optimal carbohydrate intake of elite athletes who perform high intensity practice every day, it is necessary to investigate muscle glycogen changes before and after practice to determine the amount of consumption during normal practice. In this study, we measured the muscle glycogen concentrations in thigh and upper arm muscles, and investigated the muscle glycogen changes during daily practice for two male gymnasts. Two male gymnasts (Player A and Player B) participating in an international competition were recruited for this study. The study was conducted during training camp. The glycogen contents of the thigh muscle group (the vastus lateralis and vastus intermedius muscles) of the right leg and biceps brachii muscle of the right arm were measured by carbon magnetic resonance spectroscopy. Measurements were performed three times: before the morning practice, after the afternoon practice, and before the morning practice on the following day. Body weight and body composition were measured concurrently with the three measurements of muscle glycogen concentrations. Body composition was measured using the impedance method. Meals and supplements were ingested freely during the study, and all foods and drinks were recorded. The practice routines were recorded by the investigator. To calculate energy consumption, physical activity metabolic equivalent values were determined by factor addition from practice records. The first body weight and body fat percentage were $52.6 \mathrm{~kg}, 9.7 \%$ for Player A and $61.1 \mathrm{~kg}, 10 \%$ for Player B. The carbohydrate intake per body weight was $5.6 \mathrm{~g} / \mathrm{kg}$ body weight for Player A and $6.8 \mathrm{~g} / \mathrm{kg}$ body weight for Player B. Assuming that the muscle glycogen concentration in the first measurement was $100 \%$, the muscle glycogen concentration of the thigh in the third measurement increased by $12.4 \%$ for Player A and $25.3 \%$ for Player B. Conversely, in the biceps, muscle glycogen concentrations decreased by $8.7 \%$ and $3.4 \%$ in Players A and $\mathrm{B}$, respectively. Thus, the thigh and arm muscles showed different changes in muscle glycogen. In conclusion, when assessing the optimal carbohydrate intake from the evaluation of muscle glycogen changes during normal practice in male gymnasts, we propose combined measurement of the thigh and arm.

\section{The Effect of an Eight-Week High $n-3$ and Low $n-6$ PUFA Dietary Intervention, on Submaximal and Peak Oxygen Consumption in Trained Cyclists}

\section{J. Hale ${ }^{1}$, G. Davison ${ }^{1}$, L. Passfield ${ }^{1,2}$, B. Gyimah ${ }^{3}$, M. R. Lindley ${ }^{4}$}

${ }^{1}$ School of Sport and Exercise Sciences, University of Kent, UK

${ }^{2}$ Faculty of Kinesiology, University of Calgary, Canada

${ }^{3}$ Centre for Analytical Science, Department of Chemistry, Loughborough University, UK

${ }^{4}$ School of Sport, Exercise and Health Sciences, Loughborough University, UK

This study investigated the effect of an eight-week dietary intervention designed to increase $n$-3 PUFA consumption and reduce dietary intake of $n$-6 PUFA on $\mathrm{VO}_{2 \text { peak }}$ and oxygen consumption $\left(\mathrm{VO}_{2}\right)$ during submaximal exercise. Fourteen trained male cyclists were randomly assigned to two independent groups. The experimental group received $n$-3 PUFA supplementation (2600mg EPA and 1800mg DHA per day) and followed a dietary intervention designed to reduce $n-6$ PUFA intake. The placebo group received organic soy oil and a placebo dietary intervention that 
maintained their habitual daily $n-6$ PUFA intake. Venous blood samples were obtained pre supplementation, at week four and at week eight of the dietary intervention to determine erythrocyte incorporation of $n-3$ and $n-6$ PUFA. Pre and post the dietary intervention, participants completed $4 \times 7$ minutes of submaximal cycling on a Lode ergometer at four discontinuous incremental power outputs $(100 \mathrm{~W}, 125 \mathrm{~W}, 150 \mathrm{~W}, 175 \mathrm{~W})$. Steady state oxygen consumption $\mathrm{VO}_{2}$ was assessed during each cycling bout. Following a 15-minute rest, participants performed an incremental ramp test to exhaustion (intensity increasing by $30 \mathrm{~W} \cdot \mathrm{min}^{-1}$ ) for the determination of $\mathrm{VO}_{2 \text { peak }}$. In the fish oil supplemented group, erythrocyte incorporation of total $n$-3 PUFA increased (from $10.4 \pm 2.5$ to $20.1 \pm 2.8 \%$ ) and total $n$-6 PUFA decreased (from $40.3 \pm 1.9$ to $30.8 \pm 1.3 \%$ ) over the eight-week supplementation period. There were no differences in $\mathrm{VO}_{2 \text { peak }}$ (pre 61.7 \pm 9.0 , post $61.0 \pm 7.7 \mathrm{ml} / \mathrm{kg} / \mathrm{min}$ ). During steady state submaximal cycling, there were no differences in oxygen consumption $\left(\mathrm{VO}_{2}\right)$ at any of the four power outputs in the fish oil supplemented group (e.g. $125 \mathrm{~W}$ pre $2.32 \pm 0.26$, post $1.98 \pm 0.11 \mathrm{~L} / \mathrm{min})$. In conclusion, despite the dietary intervention successfully increasing erythrocyte $n-3$ PUFA and reducing $n$-6 PUFA incorporation over an 8-week intervention period, this had no effect on oxygen consumption during submaximal exercise or $\mathrm{VO}_{2 \text { peak }}$ performance.

\section{The Effect of an Endurance Exercise Bout on Substrate Utilisation at Increasing Intensities: Characterising the FAT $_{\text {max }}$ Curve After Exer- cise With and Without Carbohydrate Ingestion}

J Bartley, S Barrows, H Strickler, C Savage, JL Areta

Research Institute for Sport and Exercise Sciences, Liverpool John Moores University, Tom Reilly Building, Byrom St Campus, Liverpool L3 3AF, UK

Substrate use shifts towards fat reliance during an endurance exercise bout, while increasing intensity makes exercise carbohydrate dependent, but the dynamics of substrate use at increasing intensities after prolonged endurance exercise are poorly charactersied. The aim of this study was to determine maximal fat utilisation (MFO) and the intensity at which MFO occurred $\left(\mathrm{FAT}_{\max }\right)$ before and after endurance exercise undertaken with and without carbohydrate ingestion. Using a single-blind, randomised counterbalanced

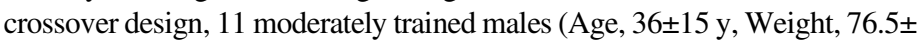
$9.5 \mathrm{~kg}, \mathrm{VO}_{2 \max }, 4.28 \pm 0.57 \mathrm{~L} \cdot \mathrm{min}^{-1}-57.3 \pm 10.0 \mathrm{ml} \cdot \mathrm{kg}^{-1} \cdot \mathrm{min}^{-1}$, Peak Aerobic Power Output, $320 \pm 36 \mathrm{~W}-4.21 \pm 0.61 \mathrm{~W} / \mathrm{kg}$ ) performed two experimental trials, where a 18-min submaximal step test $\left(\mathrm{SST}_{1} ; 3 \mathrm{~min}\right.$ at $20,33,45,58,70$ and $83 \%$ of PAPO) followed by $67-$ min cycling at $63 \%$ PAPO, finished with a second SST $\left(\mathrm{SST}_{2}\right)$. On each trial participants consumed a drink with an average of $1 \mathrm{~g} / \mathrm{CHO} / \mathrm{min}(\mathrm{CHO})$ or a volume and taste-matched placebo (PLB). Capillary blood lactate and glucose, rate of perceived exertion (RPE) and respiratory gas samples were measured throughout. Blood glucose and lactate $(P=0.012)$ were significantly higher during $\mathrm{CHO}$. Accordingly, during $\mathrm{SST}_{2}$ in PLB, absolute rates of fat oxidation increased for all intensities $(P=0.036)$ compared to $\mathrm{SST}_{1}$, MFO significantly increased by $65 \%(P<0.001)(0.49 \mathrm{v} 0.81 \mathrm{~g} / \mathrm{min})$, respiratory exchange ratio $(\mathrm{RER})$ was significantly reduced across each intensity $(P=0.038)$, and $\mathrm{FAT}_{\max }$ occurred at a higher percentage of PAPO $(49 \mathrm{v} 45 \%)(P=0.109)$. Whereas, $\mathrm{CHO}$ feeding suppressed the increase in MFO and $\mathrm{FAT}_{\max }$. No differences in RPE were observed between SST's and conditions. At higher intensities (i.e. 70 - 823\% PAPO) exercise became significantly more fat dependent in the PLB condition $(P=0.006)$. In conclusion, prolonged cycling increased MFO and $\mathrm{FAT}_{\max }$ making high exercise intensities less carbohydratedependent, whereas $\mathrm{CHO}$ ingestion suppressed MFO compared to the placebo condition following prolonged exercise.

\section{Assessing Body Fat Percentage in Professional Jockeys Using DXA} and Skinfold Prediction Equations

A Dunne ${ }^{1}$, GD Warrington ${ }^{2}$, S O'Connor ${ }^{3}$, A McGoldrick ${ }^{4}, \mathrm{~J} \mathrm{Pugh}^{4}$, M Harrison ${ }^{1}$, G O'Loughlin ${ }^{4}$, SJ Cullen ${ }^{1}$
${ }^{1}$ Waterford Institute of Technology, Waterford, Ireland

${ }^{2}$ University of Limerick, Limerick, Ireland

${ }^{3}$ Dublin City University, Dublin, Ireland

${ }^{4}$ Turf Club, The Curragh, Kildare, Ireland

Horse racing jockeys are required to maintain a low body mass almost on a daily basis throughout the racing season. Thus, the assessment of body composition for these unique weight category athletes provides an important health and performance indicator. Dual-energy X-ray absorptiometry (DXA) is regarded as the gold standard for body composition assessment but accessibility and radiation regulations limit the frequency it can be used compared to field-based methods such as skinfold thickness. The aim of the study was to assess the accuracy and variability of skinfold prediction equations at estimating percentage body fat $(\% \mathrm{BF})$ relative to DXA in a group of professional jockeys. Seventy-three professional male jockeys (Flat $\mathrm{n}=35$, and National Hunt $\mathrm{n}=38$ ) participated in a body composition assessment via DXA and skinfold thickness assessed using ISAK guidelines. Hydration status was assessed to determine if participants were euhydrated using urine specific gravity (Usg). Male specific prediction equations (Durnin and Womersley (D\&W), Evans, Lohman, Reilly, Withers and Zemski) were selected to estimate \%BF. Spearman's correlation was used to assess the relationship between DXA and the prediction equations. Descriptive characteristics for the jockey cohort were (mean \pm SD): age $27.6 \pm 7.7$ years, body mass $61.0 \pm 6.2 \mathrm{~kg}$, height $171.1 \pm 6.2 \mathrm{~cm}$ and sum of 8 skinfolds $46.3 \pm 10.6 \mathrm{~mm}$. Mean hydration status was $1.018 \pm 0.010$ Usg. Estimated \%BF was reported for DXA (14.8), Zemski (10.1), Reilly (9.2), Evans (8.1), Lohman (8.9), Withers (6.9) and D\&W (7.1). All the equations showed moderate positive correlations ( $\mathrm{r}$ value from 0.505 to $0.688, \mathrm{p}<0.01$ ) with DXA, but the \% difference between each equation and DXA ranged from $32 \%$ to $53 \%$. The selected prediction equations showed low levels of accuracy by underestimating \% BF compared to DXA in a group of professional jockeys. These findings suggest high levels of variability when using multiple methods to estimate \%BF. Therefore, caution must be taken when providing feedback to professional jockeys or if making comparisons with data obtained from the current prediction equations. If DXA is employed as a reference method, it is recommended that the sum of 8 skinfolds is used to track changes in body fat or jockey specific prediction equations are created for estimating \% BF in jockeys.

\section{Magnesium Status in Elite Track \& Field Athletes: An 8-year Analysis} of the British Athletics World Class Performance Team

SC Killer ${ }^{1,2,3}$, R Chakraverty ${ }^{1,4}$, I Taylor I ${ }^{5}$ and N Pollock ${ }^{1,6}$

${ }^{1}$ British Athletics, National Performance Institute, Athletics Centre, Loughborough University, Loughborough, UK

${ }^{2}$ School of Sport, Exercise and Health Sciences, Loughborough University, Loughborough, UK

${ }^{3}$ Institute of Sport and Physical Activity Research, University of Bedfordshire, Bedfordshire, UK

${ }^{4}$ The Football Association, St George's Park, UK

${ }^{5}$ National Centre for Sport \& Exercise Medicine, Loughborough University, Loughborough, UK

${ }^{6}$ Institute of Sport, Exercise and Health, 170 Tottenham Court Road, London UK

Magnesium plays a critical role in athlete health and performance. It is involved in numerous physiological mechanisms that support energy production, immune function, pain modulation, muscle function and bone health. Athletes may be susceptible to magnesium deficiency due to an increased utilisation during exercise. This study reports on the magnesium status of 192 Olympic and Paralympic athletes over the course of eight 
years. Athletes on the British Athletics World Class Performance Plan undertook blood testing for Red Cell Magnesium status. Athlete's ethnicity, sporting event, gender and history of tendon pain, muscle and bone injury were recorded. A total of 510 samples from 192 athletes were included in the study. On at least one blood test during the study time, $22 \%$ of athletes were identified as clinically deficient $(<1.19 \mathrm{mmol} / \mathrm{L})$. The average red cell magnesium concentration was $1.34 \mathrm{nmol} / \mathrm{L}$. Magnesium was significantly lower in female athletes and those with Black or MixedRace ethnicity and was higher in Throws athletes and Paralympians with Cerebral Palsy. Athletes with a history of Achilles or patella tendon pain had significantly lower magnesium levels than average. This study highlights the importance of investigating magnesium within this population to identify deficiency and mitigate against potential performance reduction or increased injury risk. Several areas for future work are identified to explore the relationship between magnesium and gender, ethnicity and tendon pain and muscle injury in athletes. Furthermore, new guidelines for magnesium status within athletics populations are proposed.

\section{Do Triathletes Follow a Proper Nutritional Strategy During Olympic Distance Triathlon?}

G Olcina, C Crespo, A Camacho-Cardeñosa, M Camacho-Cardeñosa, I Martinez-Guardado, R Timón

Sport Sciences Faculty, University of Extremadura, Spain

Nutritional strategy with regard to fluids and carbohydrate (CHO) ingestion in endurance events is well studied and disseminated. Long- and halfdistance triathletes seem to have this in mind, but do Olympic-distance triathletes pay attention to these nutritional recommendations during race day? The aim of this study was to evaluate fluids and CHO ingestion during an Olympic-distance triathlon race in non-professional triathletes and its relation with metabolism. Twenty-three male triathletes, who participated in a triathlon qualifier for the National Spanish Championship, were recruited. PRE- and POST-race blood glucose, triglycerides, haematocrit and body weight were measured. Carbohydrate (CHO) sources (liquid, gel or bar) and fluid ingestion (FI) were registered and split into three moments: within 15 ' before the swim, and during both the bike and the run segment. Sweat rate $(1 / h)$ was also calculated. T-test for comparing $\mathrm{PRE} / \mathrm{POST}$ data and bivariate Pearson correlation test for all variables were used. Triathletes ingested CHO to a total amount of $108 \pm 54 \mathrm{~g}(56 \pm 20 \mathrm{~g} / \mathrm{h})$. Energetic bar correlated with main source of $\mathrm{CHO}$ before the swim $(\mathrm{r}=$ $0.674, \mathrm{p}<0.001)$, isotonic drinks were the choice during the bike segment $(\mathrm{r}=0.863, \mathrm{p}<0.001)$ and gels during the run portion $(\mathrm{r}=0.953, \mathrm{p}<0.001)$. Participants drank a total of $1.96 \pm 0.631$ of fluids with a sweat rate of 1.76 $\pm 0.65 \mathrm{l} / \mathrm{h}$ with a moderate correlation between them $(\mathrm{r}=0.606, \mathrm{p}<0.05)$. Body weight decreased after competition (PRE 73.08 \pm 4.42 vs POST 71.40 $\pm 4.49, \mathrm{p}<0.001)$, blood glucose $(\mathrm{mg} / \mathrm{dl})$ increased from PRE $101 \pm 35$ to POST $124 \pm 42 \quad(\mathrm{p}<0.001)$. Plasma triglyceride concentration $(\mathrm{mg} / \mathrm{dl})$ increased from PRE $59 \pm 22$ to POST $81 \pm 19(\mathrm{p}<0.001)$, while haematocrit did not change. These data suggest that triathletes follow the general nutritional guidelines for these kinds of endurance events, ingesting $\mathrm{CHO}$ in proper quantity and sources and with a good pattern of liquid ingestion, which allows them to finish without reaching dehydration markers. This work was supported by the Government of Extremadura with funding from the European Regional Development Fund under grant (Ref: GR18003).

\section{Using Behaviour Science to Develop a Sport Nutrition Behavioural} Assessment Tool

\footnotetext{
MRN Bentley ${ }^{1,2}$, L Patterson $^{1}, \mathrm{~N}_{\text {Mitchell }}^{2}$, SH Backhouse ${ }^{1}$

${ }^{1}$ Carnegie School of Sport, Leeds Beckett University, UK

${ }^{2}$ English Institute of Sport, Sheffield, UK
}

An integral part of a sports nutritionist's role is the delivery of interventions to bring about positive change in the dietary behaviours of athletes. Given that an athlete's dietary behaviour is influenced by complex interactions of individual, social, and environmental factors, behaviour change interventions that target multiple levels of influence must be designed and implemented. To support the development of multifaceted and systemic sports nutrition programmes, this study utilised the Capability, Opportunity, Motivation-Behaviour (COM-B) model (Michie et al., 2011) and the Behaviour Change Technique Taxonomy v1 (Michie et al., 2013) to co-produce a Sports Nutrition Behavioural Assessment Tool. The initial 80 -item tool was developed based on previous studies involving focus groups with athletes and sport nutritionists. The tool was reviewed and refined in two phases: 1) a survey with seven behaviour change experts (mean age $=39 \pm 13.36$ ) to gauge alignment between the items and the theoretical constructs, and 2) semi-structured interviews (mean duration = 94 minutes) with six sports nutritionists (mean age $=35 \pm 9.77$ ) to explore acceptability and practicality of the tool in practice. Twenty-four items were considered to poorly represent the theoretical constructs and were subsequently removed. Interview data were analysed using thematic analysis (Braun and Clarke, 2006). Acceptability of the tool was high, and this was driven by its potential to develop professional practice through procedural transparency and enhanced awareness of human behaviour. Yet, to optimise the practicability of implementing the tool within high-performance sport four sub-themes were created, 1) Educate and train sports nutritionists in behavioural science, 2) Get buy-in from influential coaches in the sport, 3) Engage the broader high-performance system, and 4) Establish clear roles and responsibilities for sports nutritionists. For the first time, this study has used behavioural science to enhance the professional practice of sports nutritionists. Adopting a developmental approach, this study emphasises the importance of addressing the "whole system" (i.e., individual, social, and structural levels of influence) when implementing the Behavioural Assessment Tool within high-performance sport.

\section{Day 2 Posters}

\section{Wednesday 18th December 2019}

1. Recent nutritional considerations of the endurance female athlete I Marmol

2. Changes of body composition and cardiovascular function after a Mountain Ultramarathon race

L Esquius, $N$ Bonfanti, C Javierre

3. Effect of ingestion of a TRP receptor agonist on muscle function during high intensity exercise

GH Evans, J McPhee, J Robins, $S$ Yelland, E Sheader, $T$ Pocock and A $M W$ Yau

4. Nutri-Scan: prevalence of lactose intolerance in squad athletes of Olympic sports

V Oertzen-Hagemann, S von Spiegel, P Platen

5. Acute glutamine supplementation does not attenuate gastrointestinal damage during non-exertional heat stress AG Pearson, AL Booth, GL Close, JN Pugh

6. The ergogenic effect of caffeine on cycling time trial performance is not influenced by a common polymorphism in the ADORA2a gene in male cyclists: a pilot study

A Lynn, SP Ong, L McKitton, L Oliver, M Ranchordas, A Nichols

7. Collagen peptide supplementation and muscle fiber capillarization-a pilot study

M Kirmse, V Oertzen-Hagemann, $M$ de Marées, $W$ Bloch, P Platen

8. On the rebound: Symptoms of acute hyperphagia in an international level male Taekwondo athlete following chronic body mass loss. $C$ Langan-Evans, $M$ Germaine, $M$ Artukovic, SO Shepherd, GL Close, JP Morton 
9. Dietary Habits of Indian Sprint \& Middle-Distance Athletes M Khopkar

10. Investigating the relationship between rapid weight loss patterns and nutritional knowledge in amateur boxers

$M$ Owen, F Brown, CD Thake

Recent Nutritional Considerations of the Endurance Female Athlete

I Marmol

London South Bank University, London, UK

By the increase of females in sports, higher demand of research has been done to improve their physical performance and protect their health and wellbeing. This literature review is concentrated on the nutrition of the female endurance athlete (FEA). It contents the quality and quantity energy aspects in regard FEAs. The importance of energy availability (EA) among the FEA, defined as the result obtained from the dietary energy intake (EI) minus the energy expended in exercise (EEE) divided by kilograms of fat free mass (FFM), have been noticeable. High number of FEAs have shown to have low EA, caused by unintentional and intentional energy restriction and intensive physical activity. From the nutrition point of view, numerous studies have demonstrated the engagement of low energy dense diets and overall, poor nutrition status, by a high percentage of FEAs. This in turn, have been seen to disrupt metabolic and reproductive hormones, which have been associated with low bone mineral density (BMD), endothelial and immune dysfunctions. Moreover, the requirements of certain nutrients related to females and endurance sport, have been evaluated with the purpose of upgrading sport performance as same as preserving health and wellbeing of the FEA. Future research should be focus in new strategies and assessments of EA and most important, to provide more quality and quantity evidences based on the individual features of the FEA.

Changes of Body Composition and Cardiovascular Function After a Mountain Ultramarathon Race

L Esquius $^{1,2}$, N Bonfanti ${ }^{3}$, C Javierre ${ }^{1}$

${ }^{1}$ Department of Physiological Sciences, Medical School, University of Barcelona, Barcelona, Spain

${ }^{2}$ FoodLab, Faculty of Health Sciences, Universitat Oberta de Catalunya, Barcelona, Spain

${ }^{3}$ Real Madrid University School-European University of Madrid; Isabel 1 University, Spain

The practice of Mountain ultra-marathon (MUM) involves a high intensity and long-lasting physical effort, which leads to short-term body composition changes and cardiovascular adaptive mechanisms that allow the maintenance of physiological variables along the running. Therefore, MUM seems to be a single opportunity to study the physiological response of the body composition and the cardiovascular system to an extreme physical demand. Thus, the aim of this descriptive study was to evaluate the response of the cardiovascular function and the body composition after completing a MUM. Running history, physiological and anthropometric variables were measured in 139 male $\left(37,1 \pm 7,7\right.$ years, $\left.71,6 \pm 8,1 \mathrm{~kg}, 23,4 \pm 1,7 \mathrm{~kg} \cdot \mathrm{m}^{-2}\right)$ and 10 female $\left(38,3 \pm 5,2\right.$ years, $\left.57,7 \pm 8,1 \mathrm{~kg}, 21,6 \pm 1,9 \mathrm{~kg} \cdot \mathrm{m}^{-2}\right)$ ultra runners before (pre) and immediately after (post) the Ultrapirineu Trail (103 $\mathrm{km}$ and $6324 / 6293 \mathrm{~m} \mathrm{~d}+/ \mathrm{d}-)$. All athletes finished the race. The total time to complete the run was 19:36:05 $\pm 3: 22: 31$ hours and 23:40:09 $\pm 3: 07$ : 43 hours for men and women respectively. Significant differences between pre and post measurements were found in the following variables: heart rate increased by $44 \%\left(26.6 \pm 14.1\right.$ beats $\left.\cdot \mathrm{min}^{-1}, \mathrm{p}<0,0001\right)$ and systolic blood pressure decreases by $8 \%(10.8 \pm 14.6 \mathrm{~mm} \mathrm{Hg}, \mathrm{p}<0,0001)$. Body weight was reduced by $3.4 \%(2.4 \pm 6.1 \mathrm{~kg}, \mathrm{p}<0,0001)$ after the race. Besides, body temperature decreases by $1.5 \%$ and also forehead temperature $(0.73 \pm$ $\left.0.90{ }^{\circ} \mathrm{C}, \mathrm{p}<0,0001\right)$, scapular temperature $\left(0.42 \pm 0.72{ }^{\circ} \mathrm{C}, \mathrm{p}<0,0001\right)$, and quadriceps temperature $\left(0.47 \pm 0.88{ }^{\circ} \mathrm{C}, \mathrm{p}<0,0001\right)$. Finally, quadriceps perimeter and quadriceps fold decreased by $2 \%(0.95 \pm 4.6 \mathrm{~mm}, \mathrm{p}=$ $0,01)$ and by $1.5 \%(0.13 \pm 0.44 \mathrm{~mm}, \mathrm{p}=0,001)$ respectively. In conclusion, cardiovascular variables and body composition determinations showed significant changes after runners completed the MUM related to changes to lose heat and to maintain the temperature. For further confirmation and better understanding of the physiological effects of MUM events future studies of larger athlete populations are required.

Effect of Ingestion of a TRP Receptor Agonist on Muscle Function During High Intensity Exercise

GH Evans ${ }^{1}, \mathrm{~J} \mathrm{McPhee}^{2}, \mathrm{~J} \mathrm{Robins}^{3}$, S Yelland ${ }^{3}$, E Sheader ${ }^{3}$, T Pocock $^{3}$ and A M W Yau ${ }^{1}$

${ }^{1}$ Department of Life Sciences, Manchester Metropolitan University, UK

${ }^{2}$ Department of Sport and Exercise Sciences, Manchester Metropolitan University, UK

${ }^{3}$ Faculty of Biology, Medicine and Health, University of Manchester, UK

Transient receptor potential vanilloid 1 (TRPV1) and ankyrin 1 (TRPA1) nocireceptors are found in the oropharyngeal mucosa and are activated in response to ingestion of capsaicin and ginger respectively. It has been suggested that ingestion of substances containing TRP agonists may induce a reflex that results in reduced alpha motor neurone activity and could prevent the onset of exercise associated muscle cramps. The aim of this study was to assess whether ingestion of a TRP agonist influences muscle function during high intensity exercise. 9 healthy male participants $(21 \pm 1 \mathrm{y} ; 181 \pm 5 \mathrm{~cm} ; 83 \pm 6 \mathrm{~kg})$ completed two trials that involved ingestion of $50 \mathrm{~mL}$ of water or a commercially available TRP receptor agonist in a randomised manner. Participants performed maximal voluntary leg extension contractions (MVC) on a modified cycle ergometer before completing isometric contractions at 10 and $20 \%$ MVC with measurement of intramuscular electromyography (iEMG) of the vastus laterialis before the test substance was ingested. 15 minutes later, participants undertook a Wingate test and completed isometric contractions with iEMG at the same absolute target force as pre-exercise. No significant differences between trials were observed for peak power $(897 \pm 149$ v $848 \pm 163 \mathrm{~W} ; \mathrm{P}=0.105)$, time to peak power $(2153 \pm 970 \mathrm{v} 2392 \pm 1093 \mathrm{~ms} ; \mathrm{P}=0.527)$ or average power $(630 \pm 79$ v $619 \pm 91 ; \mathrm{P}=0.366)$ during the Wingate test but the power drop $(524 \pm 132$ v $461 \pm 132 \mathrm{~W} ; \mathrm{P}=0.028)$ was less when the TRP receptor agonist was ingested. A significant interaction between condition and intensity $(\mathrm{P}=0.031)$ was observed for the percentage change in motor unit potential area during the isometric contractions which was greater when water was ingested. The results of this study indicate that ingestion of a TRP agonist has little effect on muscular power during high intensity exercise but may influence the onset of fatigue by influencing the recruitment of motor units within active muscle.

\section{Nutri-Scan: Prevalence of Lactose Intolerance in Squad Athletes of Olympic Sports}

\section{Oertzen-Hagemann, S von Spiegel, P Platen}

Department of Sports Medicine and Sports Nutrition, Ruhr University Bochum, Gesundheitscampus Nord 10, 44801 Bochum, Germany

Gastrointestinal distress is known to impair performance and regeneration in sports. In addition to exercise, this may be due to dietary factors as food intolerances. Lactose intolerance (LI) is one of the most common intolerances. Up to $20 \%$ of the population of North Eastern Europe suffer from a genetic loss of the digestive enzyme lactase, which causes undigested lactose being metabolized by intestinal bacteria. These fermentation processes lead to digestive disorders e.g. abdominal pain. A reliable diagnosis is made by DNA-Seq, which is complex and expensive. Therefore, aim of the current study is to 1 . asses the prevalence of LI 
in German elite athletes and 2. compare the genetic testing with a questionnaire about symptoms as a potential simplified diagnostic tool. For the BISpproject (AZ: 070108/19-20) 122 hockey players from the national squad and football players from the 2 . Bundesliga (male: 76; female: 46; age: $19 \pm$ 4 years; height: $178 \pm 9 \mathrm{~cm}$; weight: $71 \pm 10 \mathrm{~kg}$ ) completed a questionnaire for symptoms related to LI, as well as the tolerability of various foods (also included but not mentioned: celiac disease, histamine intolerance, fructose malabsorption). Furthermore, a venous blood sample was taken to determine LCT-Genotype (GT) C-13910T. 27\% $(\mathrm{n}=33)$ of all athletes showed T/TGT that indicates no genetical LI. C/T-GT was confirmed in $44 \%(\mathrm{n}=54)$ referring to a decreased lactase activity but no genetical LI. In total $29 \%(\mathrm{n}=$ 35) showed C/C-GT and therefore own the genetic disposition of LI. 40\% (n $=14 / 35)$ athletes with T/T-GT indicated post-meal complaints compared with $48 \%(n=26 / 54)$ with C/T-GT and $49 \%(n=16 / 33)$ with C/C-GT. Furthermore, $26 \%(n=9 / 35)$ with T/T-GT declared that they do not tolerate dairy products or not sure whether they tolerate, compared with $43 \%(n=23 /$ 54) with C/T-GT and 39\% $(\mathrm{n}=13 / 33)$ with $\mathrm{C} / \mathrm{C}-\mathrm{GT}$. Blood analysis showed similar prevalence for LCT-GT distribution in athletes compared with general population, although a few more athletes showed a genetic disposition for LI. Nevertheless, complaints are equally distributed in all GTs, that could also be caused by other intolerances, because of unspecific symptoms. Furthermore, athletes' declarations on the tolerance of dairy products are not related to GT that may be due to different lactase activity independently from genetic disposition.

\section{Acute Glutamine Supplementation Does Not Attenuate Gastrointesti-} nal Damage During Non-Exertional Heat Stress

\section{AG Pearson, AL Booth, GL Close, JN Pugh}

Research Institute for Sport and Exercise Sciences, Liverpool John Moores University, Liverpool, UK

We have previously demonstrated that acute glutamine supplementation ameliorates exertional heat stress-induced gastrointestinal (GI) damage. The purpose of the present study was to examine the effects of acute glutamine supplementation on markers of GI permeability and damage, and, secondary, subjective symptoms of heat illness in response to nonexertional heat stress. In a randomised, placebo-controlled cross-over design, six recreationally active males completed two experimental trials; a placebo trial and a glutamine trial at $0.5 \mathrm{~g} \cdot \mathrm{kg}^{-1}$ body mass consumed $2 \mathrm{~h}$ before heat stress. Each experimental trial consisted of up to 60 minutes of hot water immersion at $40^{\circ} \mathrm{C}$. GI permeability was measured using ratio of lactulose to $L$-rhamnose (L:R) in serum. Plasma glutamine concentrations were measured at $2 \mathrm{~h}$ pre, post, and $1 \mathrm{~h}$ post heat stress and plasma intestinal fatty acid binding protein (I-FABP) concentrations were determined pre and post heat stress. Subjective heat illness symptoms were assessed throughout hot water immersion. Heat stress did not cause significant disturbance to GI permeability and L:R ratios were similar between glutamine $(0.029 \pm 0.011)$ and placebo $(0.025 \pm 0.009)$ conditions. Whilst heat stress induced a significant increase in plasma I-FABP concentration $(p=0.002)$, this was not attenuated by acute glutamine supplementation (glutamine: $1554 \pm 588$; placebo: $1123 \pm 611 \mathrm{pg} \cdot \mathrm{mL}^{-1}$; $p=0.312$ ). Symptoms of heat illness were high across both experimental conditions, with no effect of glutamine supplementation. Heat stress induces significant small intestinal mucosal damage and symptoms of heat illness, which are not diminished by glutamine consumption. Heat stress alone does not induce GI permeability, suggesting an alternative mechanism to heat illness symptomology. The relationship between heat stress and GI physiology remains unclear and warrants further research.

The Ergogenic Effect of Caffeine on Cycling Time Trial Performance is not Influenced by a Common Polymorphism in the ADORA2a Gene in Male Cyclists: A Pilot Study
A Lynn ${ }^{1}$, SP Ong ${ }^{1}$, L McKitton $^{1}$, L Oliver ${ }^{2}$, M Ranchordas ${ }^{2}$, A Nichols ${ }^{1}$

${ }^{1}$ Food and Nutrition Group, Sheffield Hallam University, Sheffield, S1 $1 \mathrm{WB}, \mathrm{UK}$

${ }^{2}$ Academy of Sport and Physical Activity, Sheffield Hallam University, Sheffield, S10 2BP, UK

It is well established that caffeine supplementation can enhance endurance exercise performance. However, inter-individual variability in the ergogenic effect of caffeine has been reported. This variability has not been adequately explained, but could reflect differences in habitual caffeine intake, training status, or age. Common genetic polymorphisms in genes encoding proteins involved in the metabolism and physiological actions of caffeine may also be important. A functional single nucleotide polymorphism (SNP) in the adenosine A2a receptor gene (ADORA2a; $1976 \mathrm{~T}>\mathrm{C}$; rs5751876) has been reported to influence the ergogenic response to caffeine. In women undertaking a $10 \mathrm{~min}$ time-trial, caffeine ingestion improved performance in all women with a T/T genotype $(\mathrm{n}=$ 6 ), whereas in those carrying a $\mathrm{C}$ allele, performance only improved in one of six participants. Only 10 to $20 \%$ of the population have a T/T genotype, so a lack of effect of caffeine in those with a $\mathrm{C}$ allele seems improbable. Thus, the aim of the current study was to further explore the effect of the ADORA2a 1976 T $>$ C SNP on the ergogenic effect of caffeine. Trained cyclists $(\mathrm{n}=23$; age 38 (SD 11) y, mass 71.7 (SD 12.3) $\mathrm{kg}$, BMI 22.9 (SD 2.3) kg/m², $\mathrm{VO}_{2} \max 60.7$ (SD 10.8) $\mathrm{ml} / \mathrm{kg} / \mathrm{min}$ ) completed a randomised cross-over trial. Each participant completed a $\mathrm{VO}_{2}$ max test, and $3 \times 16 \mathrm{~km}$ time trials $(1 \mathrm{x}$ familiarisation and $2 \times$ experimental trials) on a cycle ergometer. Caffeine $(3 \mathrm{mg} / \mathrm{kg})$ or placebo was consumed $60 \mathrm{~min}$ before the experimental time trials. The effect of caffeine on performance and interaction with genotype was assessed with a mixed model ANOVA. Caffeine caused a $1 \%$ decrease in time trial time $(-15.1 \mathrm{~s}, 95 \% \mathrm{CI},-24.9,-5.3 ; p=0.016)$. There was no significant interaction between ADORA2a genotype and change in $16 \mathrm{~km}$ time when participants were split into 3 genotypes (TT $(n=5), C T(n=4)$ and $\mathrm{CC}(\mathrm{n}=14) ; p=0.928)$ or grouped based on the presence of a $\mathrm{C}$ or $\mathrm{T}$ allele (TT v CT/CC combined; $p=0.715$; CC $\vee$ CT/TT combined; $p=$ 0.896). Moreover, the magnitude of the decrease in time was similar for each genotype $(\mathrm{CC}=15.6 \mathrm{~s} ; \mathrm{CT}=17.5 \mathrm{~s}$, $\mathrm{TT}=11.7 \mathrm{~s})$. In the present study there was no indication that a common functional SNP in the ADORA2a gene modifies the ergogenic effect of a moderate dose of caffeine on $16 \mathrm{~km}$ cycling time-trial performance in trained male cyclists.

\section{Collagen Peptide Supplementation and Muscle Fiber Capillarization- A Pilot Study}

M Kirmse ${ }^{1}$, V Oertzen-Hagemann ${ }^{1}$, M de Marées ${ }^{1}$, W Bloch ${ }^{2}$, P Platen $^{1}$

${ }^{1}$ Department of Sports Medicine and Sports Nutrition, Ruhr-University Bochum, Germany

${ }^{2}$ Department of Molecular and Cellular Sports Medicine, German Sport University Cologne, Germany

The muscle capillarization is essential for the delivery of oxygen and nutrients to the exercising muscle, and thus for the exercise capacity. Endostatin is the c-terminal fragment of type XVIII collagen $\alpha-1$ and closely associated with a decreased angiogenesis. Oertzen-Hagemann et al. previously reported an upregulation of XVIII collagen $\alpha-1$ after the daily intake of $15 \mathrm{~g}$ collagen peptides $(\mathrm{CP})$ combined with resistance training (RT) over 12 weeks. The purpose of this investigation was to assess the effects of 12 weeks of CP intake and RT on capillary density measured in the clinical study of Oertzen-Hagemann et al. 2019. Data from 14 young men $(25 \pm 3 \mathrm{yr}, 183 \pm 4 \mathrm{~cm}, 79.3 \pm 5.0 \mathrm{~kg})$ were included in the statistical analysis. Subjects received either $15 \mathrm{~g} \mathrm{CP}(\mathrm{COL})$ or $15 \mathrm{~g}$ placebo (PLA). In addition, all subjects were instructed to follow a standardized RT for 12 weeks. Muscle fiber cross sectional area (CSA) and capillary contact 
per muscle fiber were measured to calculate the mean capillary contact (CC, shown in number) per specific muscle fiber and a ratio of the CSA of specific muscle fibers per capillary (CSA/C, shown in $\mu^{2}$ ) as an expression of the capillary supply. Changes in the CSA of both muscle fiber types showed no differences between the groups ( $p>0.05)$. Delta values of CC showed a trend in type I muscle fibers (COL: P50: -0.2, P25: -0.4, P75: 0.4; PLA: P50: 0.7, P25: 0.5, P75: $1.1 ; \mathrm{p}=0.081)$ and a significant difference in type II muscle fibers (COL: P50: -0.2, P25: -0.6, P75: 0.4; PLA: P50: 0.8, P25: 0.1, P75: 1.1; $\mathrm{p}=0.029)$ for an increased number of capillaries per specific fiber in PLA compared with COL. The delta values of the CSA/C ratio only showed a significant difference between the groups in type II muscle fibers (COL: P50: 347, P25: 69, P75: 438; PLA: P50: -88, P25: -234, P75: 76; $\mathrm{p}=0.028$ ) indicating an increased CSA per capillary in COL compared with PLA. Due to the current findings in CC and CSA/C it could be speculated that angiogenesis was possibly affected by the daily intake of $15 \mathrm{~g}$ of $\mathrm{CP}$. The previously observed upregulation in collagen XVIII $\alpha-1$ might be an explanatory approach to these findings. However, the unaltered angiogenesis had no impact on improvements in muscle strength, which was tested in this study. Future research is needed to gain a greater understanding on the impact of $\mathrm{CP}$ on the muscle capillarization.

\section{On the Rebound: Symptoms of Acute Hyperphagia in an International Level Male Taekwondo Athlete Following Chronic Body Mass Loss}

C Langan-Evans, M Germaine, M Artukovic, SO Shepherd, GL Close, JP Morton

Research Institute for Sport and Exercise Sciences, Liverpool John Moores University, Liverpool, UK

Rebound hyperphagia is classified as the overcompensation of both body (BM) and fat (FM) masses following periods of chronic energy deficit. This phenomenon was first studied in the early 1900's and characterised during the seminal Minnesota Starvation Experiment by Ancel Keys and colleagues. Combat sport athletes represent a key population who are particularly susceptible to this condition, given they chronically lose BM for sequential competitions across their careers. The aim of this study was to examine potential acute rebound hyperphagia in a male international level Taekwondo athlete, following a chronic period of BM loss. Post competition, across a seven-day period, the athlete had their daily energy intake (EI) tracked utilising the 'Snap-n-Send' method. Body composition was assessed via Dual X-ray Absorptiometry and metabolic profile/energy deficiency were examined via resting metabolic rate (RMR). Venous blood sampling for measures of endocrine/lipid profiles in tandem with cardiac screening and psychological assessment via semi structured interviews were all collected at regular intervals. Following a pre competitive BM loss of $9.8 \mathrm{~kg}(14 \%)$, there was a rebound of $8.3 \mathrm{~kg}$ (13\%), accompanied by a $1.5 \mathrm{~kg}(15 \%)$ increase in FM. Pre competition average EI was set at $11,900 \mathrm{kcal} \cdot \mathrm{wk}-1$, which was substantially increased to $33,000 \mathrm{kcal} \cdot \mathrm{wk}-1$ in the ad libitum post competitive period. This resulted in a marked increase in RMR from baseline by over $390 \mathrm{kcal} \cdot d a y-1$ (23\%) and $650 \mathrm{kcal} \cdot$ day-1 (45\%) prior to competition. Despite rescuing a number of HPG axis hormones to baseline reference levels, this also resulted in unfavourable increases in triglyceride and total cholesterol/HDL/LDL lipid profiles. Additionally, the athlete exhibited marked hyperinsulinemia, with an increase of over $116 \mathrm{pmol} \cdot \mathrm{L}-1$ (446\%) compared to average precompetitive values. This study represents the first time a psycho-physiological assessment of rebound hyperphagia has been conducted within combat sports. The post competitive period represents a critical time in an athletes' nutritional recovery, which can result in pronounced negative health effects if neglected. Future research should examine this condition for longer time phases and across repeated periods to better understand how to address this issue.

\section{Dietary Habits of Indian Sprint \& Middle-Distance Athletes}

\section{Khopkar}

Department of Rehabilitation \& Sports Medicine, Sir. H.N. Reliance Foundation Hospital and Research Centre, Raja Ram Mohan Roy Road, Prarthana Samaj, Girgaum, Mumbai-400004, India

Nutrition is essential to optimize training adaptations, improve recovery, immunity and prevent injuries. The aim of this study was to determine the dietary habits of 13-21y old sprint \& mid-distance athletes $(n=29)$ selected for the Athletics Elite Camp, 2019. Anthropometric, body composition and training related details were recorded. Dietary intake was assessed using a 3 day diet recall to determine the quality of preduring-post training meals, junk food intake and quantity of energy and macronutrients consumed. These were further compared with total energy expenditure; calculated through predictive equations and standard literature recommendations. The mean body fat $(\%)$ for male $(\mathrm{n}=13)$ and female $(n=7)$ sprinters was found to be $13.1 \pm 2.0$ and $20.2 \pm 4.5$ and for male $(\mathrm{n}=9)$ and female $(\mathrm{n}=2)$ mid-distance athletes $11.3 \pm 2.0$ and 23 \pm 0.3 respectively. No significant difference between total energy expenditure and energy intake was observed. Sprint athletes consumed higher than recommended carbohydrates [t $(19)=3.485, \mathrm{p}=0.000$ ] with $44 \%$ athletes consuming biscuits/bread/chocolates/Indian sweets at least 3 or more times/week. The protein consumption for all athletes was within the recommended range suggesting adequate awareness regarding protein quantity. At least 50\% athletes reported poor recovery/ readiness to train which could be related to inadequate macronutrient quantity and quality pre-during-post training meals, as $17 \%$ of athletes trained fasted, $63 \%$ consumed only plain water during training and $18 \%$ consumed nothing immediately after training. Athletes should be educated on nutrient quality, quantity and timing of meals and be encouraged to consult accredited sports nutritionists to develop a customized, periodized and practical nutrition program.

\section{Investigating the Relationship Between Rapid Weight Loss Patterns and Nutritional Knowledge in Amateur Boxers}

\section{Owen, F Brown, CD Thake}

Faculty of Health and Life Science, Coventry University, Coventry, UK

Rapid weight loss practises (RWL) have become more prevalent in boxing over recent years. Despite the prevalence of such practices in higher performing athletes, the potential advantages in size and strength gained from competing in the lowest possible weight category must be balanced against the extreme risks such practices pose for health. It is unknown whether the use of such practices is related to a lack of nutritional knowledge. Following ethics approval, 65 amateur boxers (mean $\pm \mathrm{SD}$; age $23 \pm 3$ years, body mass $71 \pm 12 \mathrm{~kg}$, height $177 \pm 9 \mathrm{~cm}$ ) were recruited who met the following criteria; male, $>2$ years' experience, $>5$ contests. Boxers completed the Nutritional Knowledge Questionnaire for Athletes (NKQA -Furber, Roberts, and Roberts 2017) and Rapid Weight Loss Questionnaire (RWLQ -Artioli et al. 2010), with higher scores reflecting greater nutritional knowledge and RWL respectively. Correlations between RWL and NKQA (total scores, and nutrient-specific results) were assessed. Subgroup differences in NKQA and RWL scores were analysed between different weight categories and levels of experience using a 1-way ANOVA (SPSS). The prevalence of methods of weight-loss and boxers' preferred sources of nutritional education was analysed using Friedman's test. Total NKQA scores were 52 $\pm 13 \%$, with no difference between experience levels $(\mathrm{F}=0.981, p=0.381)$. No relationship was apparent between either total NKQA or subs-section scores and RWL $\left(\mathrm{r}^{2}=0.037, p=0.772\right)$. A significant difference was apparent for boxers' most frequently used RWL methods $\left(\chi^{2}=224\right.$; $p<0.001$ ), with 'restricting fluid intake' (86\%; mean rank 9.14) and 
'training in sweat suit' (86 \%; mean rank 8.90) most popular. The influences on boxers' weight-loss behaviours $\left(\chi^{2}=136, p=0.001\right)$ and nutritional knowledge $\left(\chi^{2}=80.5 ; p<0.001\right)$ showed significant differences between rankings, with coaches and other boxers holding the highest influence (weight-loss [mean rank] coach $=5.68$, other boxer $=5.05$; nutritional knowledge [mean rank] coach $=5.11$, other boxer 4.66 ). The current findings suggest nutritional education alone may not be an effective strategy to discourage RWL. Coaches and boxers should be educated on safe rates of weight-loss and the risks of RWL to improve current practises. 